\title{
GASTRO-CADx: a three stages framework for diagnosing gastrointestinal diseases
}

\author{
Omneya Attallah ${ }^{\text {Corresp., }}$, Maha Sharkas ${ }^{1}$ \\ ${ }^{1}$ Department of Electronics and Communication Engineering, College of Engineering and Technology, Arab Academy for Science \& Technology, \\ Alexandria, Egypt \\ Corresponding Author: Omneya Attallah \\ Email address: o.attallah@aast.edu
}

Gastrointestinal (GI) diseases are common illnesses that affect the GI tract. Diagnosing these GI diseases is quite expensive, complicated, and challenging. A computer-aided diagnosis (CADx) system based on deep learning ( $D L$ ) techniques could considerably lower the examination cost processes and increase the speed and quality of diagnosis.

Therefore, this paper proposes a CADx system called Gastro-CADx to classify several GI diseases using DL techniques. Gastro-CADx involves three progressive stages. Initially, four different CNN are used as feature extractors to extract spatial features. Most of the related work based on DL approaches extracted spatial features only. However, in the following phase of Gastro-CADx, features extracted in the first stage are applied to discrete wavelet transform (DWT) and discrete cosine transform (DCT). DCT and DWT are used to extract temporal-frequency and spatial-frequency features. Additionally, a feature reduction procedure is performed in this stage. Finally, in the third stage of the GastroCADx, several combinations of features are fused in a concatenated manner to inspect the effect of feature combination on the output results of the CADx and select the best-fused feature set. Two datasets referred to as Dataset I and II are utilized to evaluate the performance of Gastro-CADx. Results indicated that Gastro-CADx has achieved an accuracy of $97.3 \%$ and $99.7 \%$ for Dataset I and II respectively. The results were compared with recent related work. The comparison showed that the proposed approach is capable of classifying GI diseases with higher accuracy compared to other work. Thus, it can be used to reduce medical complications, death-rates, in addition to the cost of treatment. It can also help gastroenterologists in producing more accurate diagnosis while lowering inspection time. 
1

2

3

4

5

6 Omneya Attallah*, and Maha A. Sharkas

7 Department of Electronics and Communication Engineering, College of Engineering and

8 Technology Arab Academy for Science, Technology \& Maritime Transport, Alexandria, PO1029, 9 Egypt.

10

\section{GASTRO-CADx: A three stages framework for diagnosing gastrointestinal diseases}

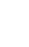

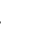

\section{Corresponding author*: Omneya Attallah}

2 Emails: o.attallah@aast.edu, 
Abstract

Gastrointestinal (GI) diseases are common illnesses that affect the GI tract. Diagnosing these GI diseases is quite expensive, complicated, and challenging. A computer-aided diagnosis (CADx) system based on deep learning (DL) techniques could considerably lower the examination cost processes and increase the speed and quality of diagnosis. Therefore, this paper proposes a CADx system called Gastro-CADx to classify several GI diseases using DL techniques. Gastro-CADx involves three progressive stages. Initially, four different $\mathrm{CNN}$ are used as feature extractors to extract spatial features. Most of the related work based on DL approaches extracted spatial features only. However, in the following phase of Gastro-CADx, features extracted in the first stage are applied to discrete wavelet transform (DWT) and discrete cosine transform (DCT). DCT and DWT are used to extract temporal-frequency and spatial-frequency features. Additionally, a feature reduction procedure is performed in this stage. Finally, in the third stage of the Gastro-CADx, several combinations of features are fused in a concatenated manner to inspect the effect of feature combination on the output results of the CADx and select the best-fused feature set. Two datasets referred to as Dataset I and II are utilized to evaluate the performance of Gastro-CADx. Results indicated that Gastro-CADx has achieved an accuracy of $97.3 \%$ and $99.7 \%$ for Dataset I and II respectively. The results were compared with recent related work. The comparison showed that the proposed approach is capable of classifying GI diseases with higher accuracy compared to other work. Thus, it can be used to reduce medical complications, death-rates, in addition to the cost of treatment. It can also help gastroenterologists in producing more accurate diagnosis while lowering inspection time.

Keywords: Gastrointestinal (GI) diseases, gastrointestinal endoscopy images, deep learning, convolution neural networks, Discrete wavelet transform (DWT), Cosine Transform. 


\section{Introduction}

62

63

64

65

66

67

68

69

70

71

72

73

74

Gastrointestinal (GI) disease is considered one of the supreme common diseases that usually infect people, causing complicated health conditions (Du et al., 2019). Based on the degree of injury, GI can approximately split into the precancerous lesion, primary GI cancer and progressive GI cancer, and benign GI diseases (Sharif et al., 2019). Among benign GI diseases are ulcers, gastritis, and bleedings which will not depreciate into cancers in short term. In contrast, precancerous GI injury could depreciate into primary GI cancer or even progressive GI cancer, in case it was not accurately diagnosed and treated in time (Du et al., 2019). Annually almost 0.7 million patients are diagnosed with gastric cancer. Since 2017, 135,430 new GI diseases arose in America. A global survey indicated that since 2017, 765,000 deaths occurred due to stomach cancer, 525,000 deaths are due to colon cancer. The poorest situations can be detected in the developing countries (e.g. the Asian countries and the Middle East) (Ali et al., 2019),(Khan et al., 2020a). Moreover, among people diseased with GI diseases, $20 \%$ of them are from China, 18\% from Brazil, 12\% from Russia, 20\% of EU, and $21 \%$ of the US (Sharif et al., 2019). The early diagnosis of GI is essential to reduce medical complications, cost of treatment, and lower death rates.

The traditional clinical method used for GI diagnosis is the intestinal biopsy of the GI tract. These biopsy samples are analyzed by medical experts using microscopes to examine the possibility of any cancerous or abnormal cells' existence. The drawbacks of such a method are being invasive and the necessity of a high degree of proficiency (Ali et al., 2019). In contrast, endoscopic imaging is a lower invasive technique for visualizing the GI tract (Kainuma et al., 2015). The endoscopic process assists the doctor in the recognition and diagnosis of gastric anomalies in their initial stages. Timely detection and diagnosis of chronic medical conditions can be healed with appropriate treatments. Hence, the imaging procedure can be very beneficial for a considerable decrease in medical complications, the cost of treatment, and death-rates, especially, the deaths that happen due to several GI cancers, which could be treated if cancer was discovered in its premalignant phase (Hamashima et al., 2015). Although, the numerous advantages of endoscopy, yet it brings along with particular trade-offs, for example, the huge number of video frames produced during the screening process of the GI tract. On average, the entire process can take from 45 minutes to 8 hours depending on the aimed GI region and the expertise of the gastroenterologist (Ali et al., 2019). The number of generated frames can reach up to 60,000 images. Most of these frames are redundant and not valuable and only a few images might have some abnormal lesions (Khan et al., 2020b). All these redundant images can be removed by examining each frame of the endoscopic video. Therefore, the manual examination of diseases through such a huge number of images is very challenging as it needs an extensive amount of time to observe the complete number of frames. Besides, at times the anomalous frames can be simply unnoticed by the gastroenterologist which can cause misdiagnosis. Therefore, such medical experts request automated schemes, that can automatically determine possible malignancies by analyzing the entire endoscopic images (Aoki et al., 2019). 
101

102

103

104

105

106

107

108

109

110

111

112

113

114

115

116

117

118

119

120

121

122

123

124

125

126

127

128

129

130

131

132

133

134

135

136

137

138

139

140

141

142

143

144

145

146

Computer-aided diagnosis (CADx) are systems utilized for automatic diagnosis of several diseases within various parts of the human body like the brain (Attallah, Sharkas \& Gadelkarim, 2020),(Attallah, Sharkas \& Gadelkarim, 2019), breast (Ragab, Sharkas \& Attallah, 2019), lung (Attallah, Ragab \& Sharkas, 2020), etc. Along with these diseases, CADx has been commonly used to diagnose GI disease in the intense by analyzing endoscopic images (Khan et al., 2020b). Such CADx has several advantages from which the patients, gastroenterologists, and medical students can benefit. These include; the reduction in the examination time of the whole endoscopic frames. Besides, the decrease in the cost of treatment as the lesion will be detected in an early phase. Moreover, CADx will improve the accuracy of the diagnosis of GI diseases compared to manual examination. Also the inspection time from endoscopic images is to be decreased. Furthermore, it may be used for training medical staff and students without the necessity of an expert (Ali et al., 2019).

In a CADx scheme, the diagnosis is carried out using each frame depending on the significant features taken out from the image. Thus, feature extraction is the key step in an accurate diagnosis of medical conditions (Attallah, 2020) like GI diseases (Khan et al., 2020b), (Ali et al., 2019), (Khan et al., 2019). Several features are calculated using handcrafted techniques in the literature like color-based, texture-based, and some others (Khan et al., 2020b), (Ali et al., 2019). Karargyris et al.(Karargyris \& Bourbakis, 2011) utilized geometric and texture features extracted from SUSAN edge detector and Gabor filter extraction methods to detect small bowel polyps and ulcers. On the other hand, Li et al (Li \& Meng, 2012a) used the uniform local binary pattern (LBP) and discrete wavelet transform (DWT). They employed an SVM classifier to detect abnormal tissues. In the same way, the authors in (Li \& Meng, 2012b) detected tumors in the intestine using DWT and LBP. Instead, Yuan et al. (Yuan \& Meng, 2014) fused the saliency map with the Bag of Features $(\mathrm{BoF})$ technique to identify polyps in endoscopic images. Initially, the authors employed the BoF method to describe the local features by using a scale-invariant feature transform (SIFT) feature vectors using k-means clustering. Next, the saliency map histogram method was utilized to extract salience features. Lastly, both features are combined and utilized to learn an SVM classifier. Later the same authors (Yuan, Li \& Meng, 2015) added the complete LBP (CLBP), LBP, uniform LBP (ULBP), and histogram of oriented gradients (HoG) features along with SIFT features to extract additional distinctive texture features. Alternatively, color-based features were extracted in (Ghosh, Fattah \& Wahid, 2018),(Deeba et al., 2018) for bleeding detection.

Recently, the advancement of deep learning (DL) methods has delivered new opportunities to improve the analysis of endoscopic images. CNNs are the most type of networks used in endoscopy (Alaskar et al., 2019). These networks can be used as classifiers or/and feature extractors. Feature extraction methods based on DL techniques have been extensively utilized in the literature. (Ghatwary, Zolgharni \& Ye, 2019),(Kim, Cho \& Cho, 2019), (Lee et al., 2019). The authors of (Khan et al., 2020a) proposed a CADx system to detect ulcers and bleeding GI diseases. Their system extracted deep features from two different layers of VGG-16 CNN. Afterward, these features were fused, and then significant features were selected using an evolutionary search method called PSO. These features were then used to train the SVM classifier. Igarashi et al, (Igarashi et al., 2020) proposed a CADx framework to classify several GI diseases using AlexNet. First, AlexNet extracted spatial features and then classified them into 14 different diseases. The authors of (Alaskar et al., 2019) proposed a DL-based CADx that utilized AlexNet and GoogleNet for ulcer detection from low contrast endoscopic videos (WEV). Features extracted from these

Peer] Comput. Sci. reviewing PDF | (CS-2020:10:54985:1:1:NEW 20 Jan 2021) 
147

networks were classified using the fully connected layer of each network separately. AlexNet was also used in (Fan et al., 2018) to detect both erosions and ulcers that are observed in the intestine. He et al. in (He et al., 2018) introduced a framework based on two cascaded CNNs. The first network is VGG-16 CNN which was used for edge detection, whereas the second is the Inception CNN which was used for classification. Similarly, Khan et al in (Khan et al., 2020b) used two CNNs, the first one is Recurrent CNN for segmentation, whereas, the second was ResNet and was used for classification. The authors in (Yuan \& Meng, 2017) suggested the use of an image manifold with stacked sparse auto-encoder to recognize polyps in endoscopic images. Instead, the authors in (Pei et al., 2017) proposed a CADx system to recognize and assess the small bowel using features extracted from long short-term memory (LSTM).

Other research articles suggested the fusion of handcrafted features and DL features. Sharif et al. (Sharif et al., 2019) proposed a CADx system for classifying GI infections. The authors extracted deep features from VGG-16 and VGG-19 CNNs and fused these features with some geometric features. These fused features were then used as input to a K-nearest neighbors (KNN) classifier. Another system was presented in (Ghatwary, Ye \& Zolgharni, 2019) to detect esophageal cancer. The system fused Gabor features and Faster Region-Based CNN (Faster R-CNN). On the other hand, Billah et al. (Billah, Waheed \& Rahman, 2017) fused the color wavelet features and CNN features for detecting polyps. The combined features were used later to fed an SVM classifier. The authors in (Nadeem et al., 2018) combined features extracted from textural analysis methods such as Haralick and LBP along with VGG-16 CNN DL features. The authors used logistic regression for classification. The authors of (Majid et al., 2020) introduced a framework that combined the DCT, DWT, color-based statistical features, and VGG16 DL features for the recognition of several GI diseases. The authors used a genetic algorithm (GA) to select features using the KNN fitness function. Finally, selected features were used to train an ensemble classifier. A summary of recent related work along with their limitations is shown in Table 1

\section{Table 1}

The main aim of this work is to construct a CADx called Gastro-CADx that is capable of accurately diagnosing more GI diseases than the proposed by others Though there are various approaches to GI detection and classification in the literature, there exist some weaknesses among these methods which are summarized in table . Gastro-CADx tries to overcome the limitations found in related studies discussed in table 1 through three cascaded stages. First of all, the majority of the current methods studied the detection and classification of a few types of GI anomalies, disease, or anatomical landmark. But, our proposed Gastro-CADx is an automatic highly accurate system to classify several GI diseases and anatomical landmarks. Some of the related studies are based on small dataset or used only one dataset to test the efficiency of their classification model, while Gastro-CADx is validated using two large datasets of several GI diseases. The few articles that classified several GI diseases achieved low accuracy, not reliable, or used only one type of CNN, ,whereas, Gastro-CADx is an accurate and reliable system that used more four CNNs. This appears in the first stage, Gastro-CADx studies several CNN based methods for feature extraction from spatial domain instead of using one or two networks to benefit from the advantages of several types of CNNs. The previous studies were either based only on an end-to-end deep learning which has very high computational cost, used only spatial features extracted from CNNs or only handcrafted feature extractions, but Gastro-CADx is not based only on spatial features, but 
192

193

194

195

196

197

198

199

200

201

202

203

204

205

206

207

208

209

210

211

212

213

214

215

216

217

218

219

220

221

222

223

224

225

226

227

228

229

230

231

232

233

234

temporal-frequency and spatial-frequency features using handcrafted feature extraction methods as well not only end-to-end based DL . This appears clearly in the second stage of Gastro-CADx. It extracts handcrafted features based on textural analysis from the temporal-frequency and spatialtemporal domains using the DL features extracted in the first stage. This reduces the high computational cost of end-to-end DL techniques. Previous related studies indicated that CNN representations have improved the performance and the abstract level for the automatic detection and classification of GI diseases (Majid et al., 2020), (Khan et al., 2020b), (Yuan \& Meng, 2017). Nevertheless, the fusion of $\mathrm{CNN}$ features with handcrafted variables could enhance diagnostic accuracy (Majid et al., 2020),(Shi et al., 2018). Therefore, in the third stage, a fusion process is introduced which combines the second stage features to benefit from the spatial, temporalfrequency, and spatial-frequency features. This stage can confirm the capacity of every feature abstraction method to mine significant information that might be disregarded from the other method. It can also reduce the computational cost compared to end-to-end DL methods.

, the previous contributions are summarized to:

- Proposing an automatic and accurate CADx system called Gastro-CADx based on three stages to classify several GI diseases and anatomical landmarks.

- The system is not based only on spatial features, but temporal-frequency and spatialfrequency features using handcrafted feature extraction methods as well.

- In the first stage, Gastro-CADx studies several CNN based methods for feature extraction from spatial domain instead of using one or two networks to benefit from the advantages of several types of CNNs.

- In the second stage, Gatro-CADx extracts handcrafted features based on textural analysis from the temporal-frequency and spatial-temporal domains using the DL features extracted in the first stage.

- Also, in the second stage, Gastro-CADx tries to minimize the problem of computational time using only reduced dimensions of features.

- In the third stage, a fusion process is introduced which combines the second stage features to benefit from the spatial, temporal- frequency, and spatial-frequency features.

- The third stage can confirm the capacity of every feature abstraction method to mine significant information that might be disregarded from the other method.

- Gastro-CADx is validated using two large datasets of several GI diseases.

- Creating an accurate automatic diagnostic system that is reliable compared to related CADx systems

\section{Material and Methods \\ 2.1 Dataset Description}

This paper employs two datasets to evaluate the performance of Gastro-CADx. The first dataset used in this paper is called Kvasir (Pogorelov et al., 2017), and denoted as Dataset I. It consists of 4000 images containing eight different GI classes . Three classes demonstrating anatomical landmarks, three demonstrating pathological states, and two associated with lesion-removal. The three anatomical landmark categories are pylorus, z-line, and cecum. The three diseased states are esophagitis, polyps, and ulcerative colitis. The two classes associated with lesion removal are dyed 
235

lifted polyps and dyed resection margins. The images are of different sizes from $720 \times 576$ up to $1920 \times 1072$ pixels. Some of these images include a green region illustrating the location and shape of the endoscope within the intestine. This information may be significant for later investigations (thus included) but must be wielded with care for the detection of the endoscopic findings. Figure 1 shows different image samples of different GI diseases.

Figure 1.

The second dataset is called HyperKvasir (Borgli et al., 2020) and named Dataset II. The images and videos of this dataset were acquired using standard endoscopy equipment from Olympus (Olympus Europe, Germany) and Pentax (Pentax Medical Europe, Germany) at a Norwegian hospital from 2008 to 2016 . The dataset consists of 10,662 labeled images and 23 classes. These classes are unbalanced; therefore, we chose only 10 balanced classes to construct Gastro-CADx. Four classes demonstrating anatomical landmarks, three demonstrating pathological states, one demonstrating quality of mucosal views, and two associated with lesion-removal. The three anatomical landmark categories are pylorus, z-line, pylorus, and cecum. The three pathological states are esophagitis, polyps, and ulcerative colitis. The two classes associated with lesion removal are dyed lifted polyps and dyed resection margins. The one demonstrating the quality of mucosal views is bowel quality. Figure 2 shows samples of images included in the dataset.

Figure 2.

\subsection{Deep Convolutional Neural Networks Architectures}

The popular type of DL approaches that is generally utilized for solving image-related classification problems in the health informatics field is the convolutional neural network (CNN) (Jin et al., 2020). In this paper, four CNNs are utilized including; AlexNet, ResNet-50, DarkNet19, and DenseNet-201 constructions. . As it can be noticed from table 1 that most related studies used AlexNet, ResNet and VGG CNNs. We did not use VGG as it has very high computational cost and number of parameters. Also, the features extracted from this network is of very huge size (Bi et al., 2020),(Ertosun \& Rubin, 2015),(Su et al., 2020). Although, AlexNet is one of the oldest architectures, but it is still being used due to its acceptable performance. This is because it has efficient computation ability and performs well with color images like these used in this paper well (Wang, Xu \& Han, 2019). We employed more recent CNNs like DarkNet and DenseNet architectures To our own knowledge darknet was not used in the literature, whereas, only few articles used DenseNet for classifying GI diseases but has several drawbacks in their proposed methods. Therefore, we used these two new CNNs architectures to test their performance and ability to classify multiple GI diseases from endoscopic images. This paragraph is added to the deep convolutional neural network architecture section page 7 in the tracked version.

The size of the input and output layers of the four networks employed used in the proposed method is shown in Table 2.

Table 2 
276

277

278

279

280

281

282

283

284

285

286

287

288

289

290

291

292

293

294

295

296

297

298

299

300

301

302

303

304

305

306

307

308

309

310

311

312

313

314

315

316

317

318

The structure of AlexNet CNN was presented in 2012 by (Krizhevsky, Sutskever \& Hinton, 2012). This construction won the ImageNet Large-Scale Visual Recognition Challenge in 2012. The structure of AlexNet includes 23 layers corresponding to 5 convolutional layers, 5 rectified linear unit (ReLu) layers, 2 normalization layers, 3 pooling layers, 3 fc layers, a probabilistic layer using softmax units, and a classification layer ending in 1000 neurons for 1000 categories (Attallah, Sharkas \& Gadelkarim, 2020).

\subsubsection{DarkNet-19}

DarkNet was first introduced in 2017 by Redmon and Farhadi (Redmon \& Farhadi, 2017). DarkNet19 is a CNN that is utilized as the spine of YOLO-V2. It commonly employs $3 \times 3$ filters and pairs the number of channels after each pooling stage. DarkNet-19 utilizes global average pooling to perform classifications in addition to $1 \times 1$ filters to reduce the feature demonstration between $3 \times 3$ convolutions. Batch Normalization is applied to regularize the classification model batch, make the training process more stable, and accelerate convergence. Darknet-19 consists of 19 convolutional layers and 5 maxpooling layers.

\subsubsection{ResNet-50}

ResNet architecture was first introduced in 2016. The essential constructing block of the ResNet is the residual block which was suggested by He et al. (He et al., 2016). The residual block offers shortcuts associations within the convolution layers, which can assist the network to step some convolution layers at a time. In other words, the residual block recommends two choices, it may attain a set of functions on the input, or it can permit this stage. Therefore, ResNet construction is supposed to be more effective than other CNNs such as AlexNet and GoogleNet as stated in(Attallah, Sharkas \& Gadelkarim, 2020). In this study, ResNet-50 is used which consists of 49 convolutional layers and one fc layer.

\subsubsection{DenseNet-201}

The latest research has revealed that CNNs can be significantly deeper, more accurate, and effective to learn when they consist of smaller connections between layers near the input and those adjacent to the output. This finding motivated Huang et al. (Huang et al., 2017) in 2017 to propose the Dense Convolutional Network (DenseNet). DenseNet joins every layer to each other layer in a feed-forward manner. While conventional CNNs with M layers have M connections - one amid every layer and its succeeding layer, DenseNet has $M(M+1) / 2$ straight connections. For every layer, the feature-maps of all previous layers are utilized as inputs, and its feature-maps are utilized as inputs into all following layers. DenseNet has numerous benefits such as their ability to lessen the vanishing-gradient issue, reinforce feature dissemination, boost feature reprocesses, and considerably decrease the number of parameters. In this paper, DenseNet-201 is used which has 201 layers deep.

\subsection{Proposed Gastro-CADx}

An efficient hybrid CADx system called Gastro-CADx is proposed to classify several GI classes from endoscopic images. Gastro-CADx involves three steps including, the image preprocessing step, followed by feature extraction, reduction and fusion step, and finally a classification step. Initially, several augmentation processes are utilized to raise the number of images in the datasets. Also, images are resized. In the feature extraction, reduction, and fusion step, three stages are performed to construct Gastro-CADx. In the first stage, valuable deep features are extracted from four CNNs including (ResNet-50, AlexNet, DenseNet-201, and DarkNet-19). In the second stage, two 
319

320

321

322

323

324

325

326

327

328

329

330

331

332

333

334

335

336

337

338

339

340

341

342

343

344

345

346

347

348

349

350

351

352

353

354

355

356

357

358

359

360

handcrafted features are used to extract features from the spatial DL features extracted in the first stage. These handcrafted features are textural analysis based features representing temporalfrequency and spatial-frequency features. The dimension of these extracted features is reduced in this stage. Afterward, is the third stage of the Gastro-CADx, where several reduced features are fused in a concatenated manner. Finally, is the classification step in which machine learning classifiers are used to identify several GI classes. Figure 3 represents the block diagram of GastroCADx.

Figure 3

\subsubsection{Image preprocessing Step}

The endoscopic images of both datasets are resized according to the size of the input layer of each CNN (Attallah, Ragab \& Sharkas, 2020) shown in table 2. Subsequently, these frames are augmented. The augmentation process is essential to raise the number of images (Attallah, Sharkas \& Gadelkarim, 2020), (Ragab \& Attallah, 2020). This technique is performed because most likely the models which are learned with an insufficient quantity of frames may over-fit (Ravì et al., 2016) (Attallah, Sharkas \& Gadelkarim, 2020). The augmentation techniques utilized in this paper to produce new endoscopic images from the training data are flipping, translation, transformation, and rotating (Talo et al., 2019),(Attallah, Ragab \& Sharkas, 2020). Each frame is flipped and translated in $\mathrm{x}$ and $\mathrm{y}$ directions with pixel range $(-30,30)$ (Attallah, Ragab \& Sharkas, 2020). Furthermore, each endoscopic image is rotated with an angle range $(0-180)$ degrees (Ragab \& Attallah, 2020).

\subsubsection{Feature Extraction, Reduction, and Fusion Step}

Gastro-CADx is based on three stages. The first stage is the DL feature extraction stage. The second is the handcrafted feature extraction and the reduction stage. Finally is the third stage known as the fusion stage.

\subsubsection{Deep Learning Feature Extraction stage (First Stage of Gastro-CADx)}

Pre-trained CNNs trained using the endoscopic frames are used to accomplish feature extraction or classification processes. During the feature mining process, valuable DL features are mined from the CNNs. Instead of utilizing the CNNs for classification, DL variables are pulled out from the fully connected layer called "fc 7" as in (Attallah, Ragab \& Sharkas, 2020), the "global average pooling 2D layer" (fifth pooling layer), and the last average pooling layer " of the AlexNet, ResNet-50, DarkNet, and DenseNet constructions as in (Ragab \& Attallah, 2020). The DL features size are 4096, 2048, 8 or 10, and 1920 for AlexNet, ResNet-50, DarkNet-19, and DenseNet-201 respectively.

\subsubsection{Handcrafted Feature Extraction and Reduction stage (Second Stage of Gastro-CADx)}

\subsection{Handcrafted Feature Extraction}

In this stage, time-frequency and spatial-frequency features based on textural analysis are determined from the DL features extracted in the previous stage. The textural features include coefficients of discrete wavelet transform (DWT) and discrete cosine transform (DCT). Each feature method is discussed below. We employed DWT and DCT as they are popular feature extraction method based on textural analysis. One of the main benefit of DCT is its capability to spatially alter to characteristics of an image for instance discontinuities and changing frequency

Peer] Comput. Sci. reviewing PDF | (CS-2020:10:54985:1:1:NEW 20 Jan 2021) 
361

362

363

364

365

366

367

368

369

370

371

372

373

374

375

376

377

378

379

380

381

382

383

384

385

386

387

388

389

390

391

392

393

394

395

396

397

398

399

400

401

402

403

404

405

manner (Bennet, Arul Ganaprakasam \& Arputharaj, 2014). It offers time-frequency representation of an image. Also, DCT has several advantages, first of all it prevents complicated calculation and presents simplicity of execution in practical purposes. Furthermore, DCT is capable of effectively managing the phase removing problem and demonstrates a powerful energy compaction estate (Imtiaz \& Fattah, 2010),(Rashidi, Fallah \& Towhidkhah, 2012). DWT and DCT are the utmost common approach to extract textural features in the medical image processing area (Lahmiri \& Boukadoum, 2013; Srivastava \& Purwar, 2017),(Mishra et al., 2017), (Anthimopoulos et al., 2014),(Benhassine, Boukaache \& Boudjehem, 2020). Textural analysis based methods are useful in extracting texture features from images which is equivalent to simulating human visual learning procedure . it is widely used in medical image processing [30-33].

- Discrete wavelet transform (DWT) is a widely used feature extraction method. DWT examines both signals and images (Lahmiri \& Boukadoum, 2013; Srivastava \& Purwar, 2017). It offers a temporal-frequency representation of an image or signal through decomposing them with the help of a group of orthogonal basis functions (Ortho-normal). Images are of two dimensions; therefore 2-D DWT is used to decompose the image (Attallah, Sharkas \& Gadelkarim, 2019). One dimensional DWT is employed on each DL feature set distinctly which results in four groups of coefficients (Ragab \& Attallah, 2020). The four groups generated after the 1-D DWT are known as three detail coefficients, $\mathrm{CD}_{1}$, and approximation coefficients, $\mathrm{CA}_{1}$. Detail coefficients consist of the diagonal, vertical, and horizontal coefficients, correspondingly.

Discrete Cosine Transform (DCT) is frequently used to transform images into basic frequency components. It displays data as a sum of cosine functions oscillating at different frequencies (Aydoğdu \& Ekinci, 2020). Generally, the DCT is applied to the imaged features to attain the DCT coefficients. The DCT coefficients are separated into three sets, known as low frequencies called (DC coefficients), middle frequencies, and high frequencies called (AC coefficients). High frequencies characterize noise and small deviations (details). Whereas, low frequencies are associated with the brightness conditions. On the other hand, middle frequencies coefficients comprise valuable information and build the basic assembly of the image. The dimension of the DCT coefficient matrix is identical to the input DL featue (Dabbaghchian, Ghaemmaghami \& Aghagolzadeh, 2010).

\subsection{Feature Reduction}

Feature reduction is an important procedure that is commonly used in the medical field to lower the huge dimension of the feature space. This reduction will correspondingly lead to a reduction in the complexity of the classification procedure (Ragab \& Attallah, 2020), the training time of the model, and avoid overfitting (Attallah et al., 2017a),(Attallah et al., 2017b). For this reason, DWT and DCT have been employed as feature reduction procedures as well as feature extractors instead of directly using the large dimension of DL features generated in the previous step. Therefore, a 1-level of DWT is applied to each DL features. The coefficients generated are are the approximation coefficients $\mathrm{CA}_{1}$, and detail coefficients $\mathrm{CD}_{1}$ of the first decomposition level of DWT.These coefficients have half the dimension of the original DL image which enters the DWT process. By this was the dimension of feature space is reduced. The CA and CD coefficients are used separately to train SVM classifiers of the next step of Gastro-CADx.

Peer] Comput. Sci. reviewing PDF | (CS-2020:10:54985:1:1:NEW 20 Jan 2021) 
406

407

408

409

410

411

412

413

414

415

416

417

418

419

420

421

422

423

424

425

426

427

428

429

430

431

432

433

434

435

436

437

438

439

440

441

442

443

444

445

446

447

The DCT, on its own, does not reduce the data dimension; however, it shrinks most of the image information in a small number of coefficients (Dabbaghchian, Ghaemmaghami \& Aghagolzadeh, 2010). Another reduction stage is usually executed to reduce the data dimension, where some of the coefficients are chosen to develop feature vectors. In this paper, 500 DCT coefficients are generated using the zigzag procedure. After this reduction procedure, these coefficients are used separately to train SVM classifiers of the next step of Gastro-CADx.

\subsubsection{Feature Fusion (Third Stage of Gastro-CADx)}

The feature vectors generated for each of DCT and DWT coefficients are then fused in a concatenated manner to form different combinations of fused features sets which are then used to classify SVM classifiers in the next step of Gastro-CADx. For DWT, initially, the CA coefficients extracted from the DL features for every two networks are fused. Then, the CA coefficients extracted from the DL features of every three networks are fused. Next, all CA coefficients extracted from DL features of the four networks are merged. The same procedure is done for the CD coefficients. For DCT, firstly the coefficients extracted from the DL features for every two networks are fused. Then, the coefficients extracted from the DL features of every three networks are fused. Finally, the DCT coefficients extracted from DL features of the four CNNs are merged

\subsubsection{Classification Step}

In this step, the classification procedure is performed using two scenarios either by an end-to-end DL (Attallah, Ragab \& Sharkas, 2020), techniques or by using the features extracted from the three stages of Gastro-CADx. The scenarios resemble four experiments. The first scenario represents the use of the four CNNs including AlexNet, ResNet-50, DarkNet-19, and DenseNet-201 as classifiers (end to end DL process). Each pre-trained CNN is created and learned distinctly and then used as a classifier. The first scenario represents experiment I. In the second scenario, the first stage of Gastro-CADx is executed which corresponds to experiment II, where the pre-trained CNNs are applied to images, and then the DL features are extracted from each network individually. These DL features are used to learn distinct SVM classifiers. These features represent spatial information only and of a huge dimension. Therefore, in the second stage of Gastro-CADx which corresponds to experiment III, DWT and DCT feature extraction methods are applied to DL features generated from each $\mathrm{CNN}$ of the first stage of Gastro-CADx to extract temporal-frequency and spatial-frequency information. These features are utilized to train SVM classifiers individually. The problem of dimensionality reduction is considered as well in the second stage of Gastro-CADx, where a reduced set of coefficients are generated using DWT and DCT methods. These coefficients represent feature vectors that are used separately to learn three SVM classifiers. Finally, in the third stage of Gastro-CADx, the reduced features are fused to form different combinations of fused features. These different combinations are used to construct several distinct SVM classifiers The aim of this stage is to examine the influence of feature fusion on the classification accuracy and select the combination which has the highest impact on the performance of the Gastro-CADx. This stage corresponds to experiment IV. Figure 4 summarizes the four experiments of Gastro-CADx.

Figure 4

Note that SVM was chosen as it is known to be a powerful classifier. It is considered to be one of the best SVM best known methods in pattern classification and image classification (Thai, Hai \&

Peer) Comput. Sci. reviewing PDF | (CS-2020:10:54985:1:1:NEW 20 Jan 2021) 
448

449

450

451

452

453

454

455

456

457

458

459

460

461

462

463

464

465

466

467

468

469

470

471

472

473

474

475

476

477

478

479

480

481

482

483

484

485

486

Thuy, 2012). It performs well with large dimension space and of multi-class. As it uses kernel function which maps the feature space into new domain that can easily separate between classes of a dataset. Therefore, it is commonly used with the huge dimension of DL features extracted from CNNs (Ragab et al., 2019), (M.M. et al., 2017), (Zhang et al., 2020), (Das et al., 2020), (Xue et al., 2016), (Leng et al., 2016),(Wu et al., 2018), (Sampaio et al., 2011) achieving outperforming results. Also, as you can see in table 1, that SVM is the commonly used in the literature It can be observed that articles that used SVM achieved the highest performance as (Khan et al., 2020b) which achieved an accuracy of 99.13\%, (Khan et al., 2020a) achieving an accuracy of 98.4\%, (Ghatwary, Ye \& Zolgharni, 2019) obtaining an accuracy of 95\%, (Billah, Waheed \& Rahman, 2017) achieving an accuracy of $98.65 \%$

\section{Experimental Setup}

Several parameters are attuned after fine-tuning the fc layer of the CNNs. The number of epochs and the initial learning rate for the four CNNs are 10 and $10^{-4}$ respectively as in (Attallah, Sharkas $\&$ Gadelkarim, 2020). The mini-batch size and validation frequency are 10 and 3 . The weight decay and momentum are set to $5 \times 10^{-4}$ and 0.9 respectively. The optimization algorithm used is the Stochastic Gradient Descent with Momentum (SGDM). To measure the capacity of the Gastro-CADx to classify several GI diseases, 5-fold cross-validation is engaged. This means that the GI datasets are divided into $80 \%-20 \%$ for training and validation. The SVM classifiers are taught with 4 folds and verified by the remaining fold. Thus, the models are taught five times and the testing accuracy is calculated for each time then averaged. The kernel functions used for the SVM classifier are linear, quadratic, and cubic.

\section{Evaluation Performance}

The presented Gastro-CADx framework is evaluated with numerous measures for instance; F1score, precision, accuracy, sensitivity, and specificity. The formulas which are utilized in calculating such metrics are displayed below in equations (Attallah, Ragab \& Sharkas, 2020) (1$5)$. $5)$

. 
487

488

489

490

491

492

493

494

495

496

497

498

499

500

501

502

503

504

505

506

507

508

509

510

511

512

513

514

515

516

517

518

519

520

521

522

523

524

525

526

The results of four experiments of Gastro-CADx are presented in this section. Experiment I is an end to end DL process where the four CNN are employed to perform classification. In experiment II (first stage of Gastro-CADx), DL features are extracted from the four CNNs and used to train distinct SVM classifiers. Experiment III (second stage of Gastro-CADx) represents the use of the second stage of feature extraction and reduction methods which employs DCT and DWT to extract temporal-frequency and spatial-frequency information from the images. In this experiment, reduced coefficients generated from DWT and DCT methods are employed to train SVM classifiers. In experiment IV, different combinations of fused features are generated and utilized to inspect the effect of feature combination on Gastro-CADx performance.

\subsection{Experiment I Results}

The results of the end-to-end DL procedure employed for classification are illustrated in tables 3 and 4 for Dataset I and Dataset II respectively. Table 3 shows that the highest accuracy of $91.66 \%$ is achieved by ResNet-50 followed by an accuracy of $90.08 \%, 89.83 \%, 88.32 \%$ attained by DarkNet-19, DenseNet-201, and AlexNet respectively for Dataset I. Table 4 demonstrates that the peak accuracy of $94.75 \%$ is achieved by ResNet-50 followed by an accuracy of $93.26 \%$, 91.93\%,91.66\% attained by DarkNet-19, DenseNet-201, and AlexNet respectively for Dataset II.

Table 3

Table 4

\subsection{Experiment II Results}

This experiment represents the first stage of Gastro-CADx. The results of this experiment are shown in figures 5 and 6 for Dataset I and Dataset II respectively. Figure 5 indicates the maximum accuracies of $94.4 \%$ and 94.3 are attained by DarkNet-19 using linear and quadratic SVM classifiers using Dataset I. Subsequently, ResNet-50 features achieve an accuracy of 93.5\%, 93.4\%, and 93.4\% using linear, quadratic, and cubic SVM classifiers respectively. Following, AlexNet and DenseNet-201 features obtain an accuracy of 92.9\%, 93\%, 92.7\%, and 91\%. 91.5\%, $91.7 \%$ for using linear, quadratic, and cubic SVM classifiers respectively. Figure 6 shows the peak accuracies of $96.9 \%, 96.8 \%, 96.7 \%$ are achieved by ResNet-50 using linear, quadratic, and cubic SVM classifiers constructed with Dataset II. Next, DarkNet features attain an accuracy of 96.4\%, 96\%, and 95.2\% using linear, quadratic, and cubic SVM classifiers respectively. Following, AlexNet and DenseNet-201 features obtain an accuracy of 95.5\%, 95.7\%, 95.3\% and 94.7\%. $94.6 \%, 94.6 \%$ for using linear, quadratic, and cubic SVM classifiers respectively.

Figure 5

Figure 6

\subsection{Experiment III Results}

This experiment represents the second stage of Gastro-CADx. The results of this experiment are shown in figures 7-10 for Dataset I and figures 11-14 for Dataset II. Figure 7 shows the classification accuracy for the three SVM classifiers constructed with CA and CD coefficients of DWT, besides the 500 DCT coefficients extracted from the ResNet-50 CNN using Dataset I. The figure indicates that the peak accuracy of $93.6 \%$ is achieved using the 500 DCT coefficients with linear SVM. Almost the same accuracy of $93.5 \%$ is attained using the CA coefficients of DWT. 
527 Figure 7

528 Figure 8 demonstrates the classification accuracy for the three SVM classifiers built with CA and 529 CD coefficients of DWT, in addition to the 500 DCT coefficients extracted from AlexNet CNN 530 using Dataset I. The figure specifies that the highest accuracy of $93.3 \%$ is accomplished using the 531 CD coefficients of DWT with a quadratic SVM classifier. A slightly lower accuracy of $92.9 \%$ is 532 attained using the CA coefficients of DWT.

533 Figure 8

534 Figure 9 displays the classification accuracy for the three SVM classifiers constructed with CA 535 and CD coefficients of DWT, as well as the 500, DCT coefficients extracted from DenseNet CNN 536 using Dataset I. The figure identifies that the highest accuracy of $91.1 \%$ is accomplished using the 537 CA coefficients of DWT with a cubic SVM classifier. A lower accuracy of $90.6 \%$ is reached using 538 the CA coefficients of DWT with a linear SVM classifier.

539 Figure 9

540 Figure 10 shows the classification accuracy for the three SVM classifiers created with the CA and 541 CD coefficients of DWT, besides the DCT coefficients extracted from DarkNet-19 CNN using 542 Dataset I. Note that, since the number of DL features extracted from DarkNet-19 was only 8 (which 543 is already a small dimension of features), all the DCT coefficients are used in this experiment 544 without the need of the zigzag scanning procedure. The figure indicates that the highest accuracy 545 of $94.7 \%$ is accomplished using the DCT coefficients with linear SVM.

546 Figure 10

547 Figure 11 shows the classification accuracy for the three SVM classifiers constructed with CA and 548 CD coefficients of DWT, besides the 500 DCT coefficients extracted from the ResNet-50 CNN 549 using Dataset II. The figure indicates that the peak accuracy of $96.9 \%$ is achieved using the CA 550 coefficients with linear, cubic, and quadratic SVM. Almost the same accuracy of $96.8 \%$ is attained 551 using the CD coefficients of DWT with linear, cubic, and quadratic SVM and the 500 DCT 552 coefficient with linear SVM.

553 Figure 11

554 Figure 12 reveals the classification accuracy for the three SVM classifiers learned with CA and 555 CD coefficients of DWT, besides the 500 DCT coefficients extracted from AlexNet CNN using 556 Dataset II. The figure specifies that the highest accuracy of $95.6 \%$ is accomplished using the CA 557 coefficients of DWT with a quadratic SVM classifier. A slightly lower accuracy of $95.5 \%$ is 558 attained using the CD coefficients of DWT with quadratic SVM.

559 Figure 12

560 Figure 13 indicates the classification accuracy for the three SVM classifiers built with CA and CD 561 coefficients of DWT, besides the 500, DCT coefficients extracted from DenseNet CNN using 562 Dataset II. The figure identifies that the highest accuracy of $94.4 \%$ is accomplished using the CA 563 coefficients of DWT with cubic and quadratic SVM classifiers. The same accuracy is reached 564 using the CD coefficients of DWT with a quadratic SVM classifier.

565 Figure 13. 
566 Figure 14 demonstrates the classification accuracy for the three SVM classifiers constructed with 567 CA and CD coefficients of DWT, in addition to the DCT coefficients extracted from DarkNet-19 568 CNN using Dataset II. As the number of DL features mined from DarkNet-19 was only 10 in the 569 case of Dataset II (which is already a small dimension of features), all the DCT coefficients are 570 employed in this experiment without the necessity of the zigzag scanning process. The figure 571 specifies that the peak accuracy of $96.4 \%$ is obtained using the DCT coefficients with linear SVM.

572 Figure 14

573

574

575

576

577

578

579

580

581

582

583

584

585

586

587

588

589

590

591

592

593

594

595

596

597

598

599

600

601

602

603

604

605

\subsection{Experiment IV Results}

This experiment represents the third stage of Gastro-CADx. This experiment aims to explore the effect of combining features on the CADx's performance. Moreover, to search for the best combination of fused feature set which has the highest influence on the classification accuracy. To form the fused feature sets, firstly for DWT, the CD coefficients extracted from the DL features for every two CNNs are fused. Next, the CD coefficients extracted from the DL features of each three CNNs are merged. Afterward, all CD coefficients extracted from the DL features of the four CNNs are combined. A similar fusion process is executed for the CA coefficients. For DCT, initially, the coefficients extracted from the DL features for every two CNNs are fused. Afterward, the DCT coefficients extracted from the DL featured of each three CNNs are fused. Next, the coefficients extracted from DL images of the four CNNs are merged.

Table 5 displays a comparison between classification accuracy achieved using CA and CD features extracted from different combinations of the DL features generated from the four CNNs employed in Gastro-CADx using Dataset I. This comparison shows the CA features has slightly higher accuracy than CD features for all combination of fused features except for AlexNet + ResNet and AlexNet + DenseNet. The maximum performance (written in bold) is achieved using CA and CD features extracted using the fusion of AlexNet+ ResNet+DenseNet CNNs, where the highest accuracy of $97.3 \%$ is attained using CA features extracted using AlexNet+ ResNet+DenseNet CNNs with both quadratic and cubic SVM. On the other hand, Table 6 presents a comparison between classification accuracy accomplished using 500 DCT features extracted from different combinations of the DL variables produced from the four CNNs employed in Gastro-CADx using Dataset I. This comparison indicates the maximum performance (written in bold) is achieved using DCT features extracted using AlexNet+ ResNet+DenseNet+ DarkNet CNNs, where the highest accuracy of $97.3 \%$ is attained using features extracted using AlexNet+ ResNet+DenseNet + DarkNet CNNs with both quadratic and cubic SVM.

Table 5

Table 6

Table 7 demonstrates a comparison between the classification accuracy accomplished using CA and $\mathrm{CD}$ features extracted from different combinations of the DL variables produced from the four CNNs using Dataset II. This comparison indicates the CA features has slightly higher accuracy than CD features for all combinations of fused features except for AlexNet+ ResNet and AlexNet+ DenseNet. The peak performance (written in bold) is achieved using CA and CD features extracted using AlexNet+ ResNet+DenseNet CNNs, where the maximum accuracy of $99.7 \%$ is reached 
606

607

608

609

610

611

612

613

614

615

616

617

618

619

620

\section{1}

622

623

624

625

626

627

628

629

630

631

632

633

634

635

636

637

638

639

640

641

642

643

644

645

646

647

using CA features extracted using AlexNet+ResNet+DenseNet CNNs using cubic SVM. In contrast, Table 8 shows a comparison between classification accuracy accomplished using 500 DCT features extracted from different combinations of the DL variables generated from the four CNNs employed in Gastro-CADx using Dataset II. This comparison specifies the maximum accuracy (written in bold) is achieved using 500 DCT features extracted using AlexNet+ResNet+DenseNet CNNs and AlexNet+ ResNet+DenseNet+ DarkNet CNNs, where the highest accuracy of $97.3 \%$ is attained using linear, quadratic, and cubic SVM classifiers. Table 9 shows the performance metrics for cubic SVM classifiers trained with the fused CA features extracted from AlexNet+ResNet+DenseNet CNNs using Dataset I and Dataset II. The results of table 9 indicate that the specificity of 0.9959 and 0.9996 , sensitivity of 0.9715 and 0.9965 , precision of 0.9718 and 0.9961 , and F1 score of 0.9715 and 0.9963 are obtained for Dataset I and Dataset II respectively

Table 7

Table 8

Table 9

\section{Discussion}

The manual diagnosis of GI diseases with a huge number of endoscopic images is very challenging and time-consuming. Besides, at times the image containing the abnormality can be simply unobserved by the medical expert which can lead to misdiagnosis. Therefore, there is an essential need for automatic systems that have the capability to automatically identify possible anomalies by analyzing the entire endoscopic images (Aoki et al., 2019). Nowadays, with the current development of DL and imaging processing technologies, CADx systems have been frequently used to help gastroenterologists in automatically examining endoscopic images and recognizing the GI disease (Khan et al., 2020b). In this study, an automatic CADx system called Gastro-CADx is proposed. The proposed CADx involves three steps including the image preprocessing step, followed by the feature extraction, reduction, and fusion step, and finally the classification step. Primary the endoscopic images were augmented. Next, is the feature extraction, reduction, and fusion step. which presents the three stages of Gastro-CADx. In the first stage of Gastro-CADx, four spatial valuable DL features were extracted from the four CNNs and used to train SVM classifiers. Next, in the second stage of Gastro-CADx, DCT, and DWT feature extraction methods were employed to extract temporal-frequency and spatial-frequency features. These methods were used for feature reduction as well. These extracted features are utilized to construct SVM classifiers. Finally, in the third stage of Gastro-CADx, the coefficients of the DCT and DWT were fused to form different combinations of fused feature sets. This stage examined the influence of fusing features on the performance of the CADx. Besides, the third stage of Gastro-CADx searched for the greatest mixture of features that influenced Gastro-CADx's performance. Two datasets, namely Dataset I and Dataset II were used to evaluate the performance of the proposed GastroCADx.

The first stage of Gastro-CADx is compared with the end-to-end DL CNNs of experiment I and the results are shown in tables 10 for Dataset I and II. It can be observed from Table 10 that the first stage of Gastro-CADx has higher accuracies compared to the end-to-end CNNs constructed

Peer] Comput. Sci. reviewing PDF | (CS-2020:10:54985:1:1:NEW 20 Jan 2021) 
648 in experiment I for both datasets. The highest accuracy achieved in the first stage of Gastro-CADx 649 is $94.4 \%$ using linear SVM trained with DarkNet-19 features for Dataset I (written in bold). 650 Whereas, for Dataset II, the peak accuracy attained in the first stage of Gastro-CADx is $96.9 \%$ using linear SVM trained with DarkNet-19 features.

Table 10

653

654

655

656

657

658

659

660

661

662

663

664

665

666

667

668

669

670

671

672

673

674

675

676

677

678

679

680

681

682

683

684

685

686

687

688

689

690

691

It was found that most of the previous studies directly used spatial DL features to perform the classification, however in the paper we tried extracting spatial-temporal-frequency DL features using DWT and spatial-frequency DL features using DCT to examine their influence on the classification performance of Gastro-CADx (stage two of Gastro-CADx). DCT and DCT was also performed to reduce the huge dimension of the DL spatial features. It is proved from figure 15, that for dataset I, stage two has enhanced the classification performance with reduced feature set, while for dataset II it attained the same accuracy but with lower feature dimension. The second stage of Gastro-CADx has reduced the features extracted from the first stage of Gastro-CADx with almost the same accuracy but with fewer feature dimensional space for Dataset I and Dataset II. The highest accuracy of $94.7 \%$ of the second stage of Gastro-CADx for Dataset I was obtained using linear SVM classifier trained with the DCT coefficients extracted from deep learning features of DarkNet-19 CNN . Whereas, for Dataset II, the peak accuracy of $96.9 \%$ is achieved using linear SVM classifier trained with the CA coefficients extracted from deep learning features of ResNet-50 CNN.

On the other hand, the third stage of Gastro-CADx has further enhancement on the classification accuracy of Gastro-CADx as shown in figure 15 for Dataset I and Dataset II. Figure 15 shows the highest classification accuracy achieved using each stage of Gastro-CADx for Dataset I and II respectively. It can be noticed from the third stage of Gastro-CADx (experiment IV) that the fusion of DCT and DWT of DarkNet and DenseNet CNNs yielded the worst accuracy of around 47-49\% for both Dataset I and Dataset II. Whereas, the highest accuracy of $97.3 \%$ and $99.7 \%$ is achieved using cubic SVM classifier trained with the fused CA coefficients extracted using deep learning features of AlexNet+ResNet+DesneNet for Dataset I and Dataset II respectively. Table In order to make a fair comparison regarding the computational time with other related studies, we both should use the same platform and environment like using the same processor and video controller and other specifications which can vary the computational time. Since, this is very hard to be accomplished as an alternative, we compared the computational cost of the proposed Gastro-CADx with ResNet CNN (end-to-end deep learning techniques) which is widely used in the literature and achieved the highest accuracy using both Dataset I and Dataset II as shown in table 10. This comparison is shown in table 11 which compares both the classification accuracy and training time of ResNet CNN using end-to-end procedure with Gastro-CADx. Table 11 proves that GastroCADx has much lower computation time than ResNet (end-end classification) while attaining higher accuracy for both datasets. This is because the computation time for ResNet is $80,580 \mathrm{sec}$ and $100,800 \mathrm{sec}$ for Dataset I and II respectively which is much higher than the $210 \mathrm{sec}$ and 780 sec achieved by Gastro-CADx. Also, the accuracy for ResNet is $90.08 \%$ and $94.75 \%$ for Dataset I and II respectively which is much higher than the $97.3 \%$ and $99.7 \%$ obtained by Gastro-CADx Note that we also searched related studies to see if the authors have mentioned the computational time of their proposed methods, but unfortunately this information was missing.

Figure 15

Table 11

Peer] Comput. Sci. reviewing PDF | (CS-2020:10:54985:1:1:NEW 20 Jan 2021) 
692

693

694

695

696

697

698

699

700

701

702

703

704

705

706

707

708

709

710

711

712

713

714

715

716

717

718

719

720

721

722

723

724

725

726

727

728

729

730

731

732

733

734

All experiments are done with Matlab 2020 a. The processor used is Intel(R) Core(TM) i77700HQ, CPU@2.80 GHZ 2.8 GHZ, RAM 16 GB, and 64-bit operating system. The video controller is NVIDIA GeForce GTX 1050.

A comparison is made to compare the performance of Gastro-CAD with the latest relevant work that used Dataset I. The results of this assessment are displayed in table 12. Table 12 results prove the competence of Gastro-CADx compared to other previous related studies. Gastro-CADx proposed in this paper appears to perform well on all of the metrics provided in Table 12. GastroCADx outperformed the systems presented by (Ahmad et al., 2017), (Pogorelov et al., 2017) (first method), (Owais et al., 2019), and (Owais et al., 2019) as they used only spatial information extracted from one or two CNN. The proposed system also outperformed (Pogorelov et al., 2017), (Nadeem et al., 2018) as they used only handcrafted global features and did not benefit from using the spatial information of features extracted with DL techniques. Although, (Agrawal et al., 2017) combined DL features with handcrafted global features, yet their performance is lower than Gastro-CADx. This is because Gastro-CADx considered the fusion of two types of textural features while reducing the feature space.

\section{Table 12}

Dataset II is a new dataset for GI disease that was just released in 2020. Therefore, there is still no research articles to compare with. For this reason, we only compared with the ResNet-50 CNN used in (Borgli et al., 2020) as well as the other three CNNs employed in experiment I of GastroCADx and illustrated in table 13. The results of Gasto-CADx shown in table 12 verifies its competence. It outperformed the classification accuracy achieved by ResNet-50 used in (Borgli et al., 2020). Gastro-CADx has also better performance than the classification accuracy achieved by AlexNet, DenseNet-201, and DarkNet-19 CNNs. This is because Gastro-CADx extracted not only spatial features but temporal-frequency and spatial-frequency features. It also used DCT and DWT not only for feature extractors but also for feature reduction methods. Moreover, it fuses these several reduced $t$ features to enhance the performance of the CADx.

\section{Table 13}

The three stages of Gatro-CADx based on deep CNNs, DCT, and DWT showed the best performance with the highest accuracies of $97.3 \%$ and $99.7 \%$ for Dataset I and Dataset II respectively. The following article (Attallah, 2020) (Colquhoun, 2014), that that the reliability of a medical system requires that the sensitivity should be greater than or equivalent to $80 \%$, the specificity is greater or equivalent to $95 \%$, and the precision is more or equivalent to $95 \%$. The specificities, sensitivities, and precision shown in table 9 are all larger than 95\%, therefore GastroCADx can be considered a reliable system. This remarkable reliability and performance of GastroCADx rises its usability in the diagnosis of several GI diseases by automatically detecting several types of GI lesions or anomalies. Our AI-based Gastro-CADx framework can help the medical experts in an effective diagnosis of several complex GI diseases. Furthermore, it may assist gastroenterologists in reaching a more accurate diagnosis whereas reducing examination time. The proposed system can be used to decrease medical obstacles, death-rates in addition to the cost of treatment.

\section{Conclusion}

Peer] Comput. Sci. reviewing PDF | (CS-2020:10:54985:1:1:NEW 20 Jan 2021) 
735

736

737

738

739

740

741

742

743

744

745

746

747

748

749

750

751

752

753

754

755

756

757

758

759

760

761

762

763

764

765

766

767

768

769

770

771

772

773

774

775

776

This paper introduced a CADx system called Gastro-CADx for the automatic classification of GI diseases based on DL techniques. Gastro-CADx consist of three stages. The first stage is based on DL feature extraction techniques to extract spatial information from endoscopic images. The second stage extracted some temporal-frequency and spatial-frequency features. The feature reduction procedure is also considered in this stage. The third stage is a feature fusion based process where several features sets extracted in the second stage are fused to form numerous combinations of fused features. The results of the three stages of Gastro-CADx verified that the proposed system was capable of accurately classifying GI diseases. The first stage of GastroCADx achieved higher accuracy than that of end to end DL CNNs. Moreover, the results of the second stage of Gastro-CADx indicated that using the temporal-frequency and spatial-frequency has a better performance compared to using only spatial features. Besides, the second stage of Gastro-CADx achieved competitive performance to the first stage with a lower dimension of features. Also, the third stage has further improvement in the performance of Gastro-CADx which indicated that feature fusion had a significant impact on the accuracy of classification. The performance of the Gastro-CADx is competitive with recent related work based on the same dataset. This means the proposed method can be used efficiently for the diagnosis and classification of GI diseases. Consequently, the cost of medical investigations, medical complications, and death-rates will be reduced. Moreover, the quality of diagnosis will be enhanced as well as the accuracy. Future work will focus on combining multiple datasets to form a multicenter study. Besides, exploring more $\mathrm{CNNs}$ and more handcrafted feature extraction methods.

\section{References}

Agrawal T, Gupta R, Sahu S, Espy-Wilson CY. 2017. SCL-UMD at the Medico Task-MediaEval 2017: Transfer Learning based Classification of Medical Images. In: MediaEval.

Ahmad J, Muhammad K, Lee MY, Baik SW. 2017. Endoscopic image classification and retrieval using clustered convolutional features. Journal of medical systems 41:196.

Alaskar H, Hussain A, Al-Aseem N, Liatsis P, Al-Jumeily D. 2019. Application of convolutional neural networks for automated ulcer detection in wireless capsule endoscopy images. Sensors 19:1265.

Ali H, Sharif M, Yasmin M, Rehmani MH, Riaz F. 2019. A survey of feature extraction and fusion of deep learning for detection of abnormalities in video endoscopy of gastrointestinal-tract. Artificial Intelligence Review:1-73.

Anthimopoulos M, Christodoulidis S, Christe A, Mougiakakou S. 2014. Classification of interstitial lung disease patterns using local DCT features and random forest. In: 2014 36th Annual International Conference of the IEEE Engineering in Medicine and Biology Society. IEEE, 6040-6043.

Aoki T, Yamada A, Aoyama K, Saito H, Tsuboi A, Nakada A, Niikura R, Fujishiro M, Oka S, Ishihara S. 2019. Automatic detection of erosions and ulcerations in wireless capsule endoscopy images based on a deep convolutional neural network. Gastrointestinal endoscopy 89:357-363.

Attallah O. 2020. An Effective Mental Stress State Detection and Evaluation System Using Minimum Number of Frontal Brain Electrodes. Diagnostics 10:292-327. 
777

778

779

780

781

782

783

784

785

786

787

788

789

790

791

792

793

794

795

796

797

798

799

800

801

802

803

804

805

806

807

808

809

810

811

812

813

814

815

Attallah O, Karthikesalingam A, Holt PJ, Thompson MM, Sayers R, Bown MJ, Choke EC, Ma X. 2017a. Feature selection through validation and un-censoring of endovascular repair survival data for predicting the risk of re-intervention. BMC medical informatics and decision making 17:115-133.

Attallah O, Karthikesalingam A, Holt PJ, Thompson MM, Sayers R, Bown MJ, Choke EC, Ma X. 2017b. Using multiple classifiers for predicting the risk of endovascular aortic aneurysm repair re-intervention through hybrid feature selection. Proceedings of the Institution of Mechanical Engineers, Part H: Journal of Engineering in Medicine 231:1048-1063.

Attallah O, Ragab DA, Sharkas M. 2020. MULTI-DEEP: A novel CAD system for coronavirus (COVID-19) diagnosis from CT images using multiple convolution neural networks. PeerJ 8:e10086.

Attallah O, Sharkas MA, Gadelkarim H. 2019. Fetal Brain Abnormality Classification from MRI Images of Different Gestational Age. Brain Sciences 9:231-252.

Attallah O, Sharkas MA, Gadelkarim H. 2020. Deep Learning Techniques for Automatic Detection of Embryonic Neurodevelopmental Disorders. Diagnostics 10:27-49.

Aydoğdu Ö, Ekinci M. 2020. An Approach for Streaming Data Feature Extraction Based on Discrete Cosine Transform and Particle Swarm Optimization. Symmetry 12:299.

Benhassine NE, Boukaache A, Boudjehem D. 2020. Medical Image Classification Using the Discriminant Power Analysis (DPA) of Discrete Cosine Transform (DCT) Coefficients. In: Real Perspective of Fourier Transforms. IntechOpen,.

Bennet J, Arul Ganaprakasam C, Arputharaj K. 2014. A discrete wavelet based feature extraction and hybrid classification technique for microarray data analysis. The Scientific world journal 2014.

Bi Z, Yu L, Gao H, Zhou P, Yao H. 2020. Improved VGG model-based efficient traffic sign recognition for safe driving in 5G scenarios. International Journal of Machine Learning and Cybernetics:1-12.

Billah M, Waheed S, Rahman MM. 2017. An automatic gastrointestinal polyp detection system in video endoscopy using fusion of color wavelet and convolutional neural network features. International journal of biomedical imaging 2017.

Borgli H, Thambawita V, Smedsrud PH, Hicks S, Jha D, Eskeland SL, Randel KR, Pogorelov K, Lux M, Nguyen DTD. 2020. HyperKvasir, a comprehensive multi-class image and video dataset for gastrointestinal endoscopy. Scientific Data 7:1-14.

Colquhoun D. 2014. An investigation of the false discovery rate and the misinterpretation of pvalues. Royal Society open science 1:140216.

Dabbaghchian S, Ghaemmaghami MP, Aghagolzadeh A. 2010. Feature extraction using discrete cosine transform and discrimination power analysis with a face recognition technology. Pattern recognition 43:1431-1440.

Das D, Mahanta LB, Baishya BK, Ahmed S. 2020. Classification of Childhood Medulloblastoma and its subtypes using Transfer Learning features-A Comparative Study of Deep 
816

817

818

819

820

821

822

823

824

825

826

827

828

829

830

831

832

833

834

835

836

837

838

839

840

841

842

843

844

845

846

847

848

849

850

851

852

853

854

855

Convolutional Neural Networks. In: 2020 International Conference on Computer, Electrical \& Communication Engineering (ICCECE). IEEE, 1-5.

Deeba F, Islam M, Bui FM, Wahid KA. 2018. Performance assessment of a bleeding detection algorithm for endoscopic video based on classifier fusion method and exhaustive feature selection. Biomedical Signal Processing and Control 40:415-424.

Du W, Rao N, Liu D, Jiang H, Luo C, Li Z, Gan T, Zeng B. 2019. Review on the applications of deep learning in the analysis of gastrointestinal endoscopy images. IEEE Access 7:142053142069.

Ertosun MG, Rubin DL. 2015. Probabilistic visual search for masses within mammography images using deep learning. In: 2015 IEEE International Conference on Bioinformatics and Biomedicine (BIBM). IEEE, 1310-1315.

Fan S, Xu L, Fan Y, Wei K, Li L. 2018. Computer-aided detection of small intestinal ulcer and erosion in wireless capsule endoscopy images. Physics in Medicine \& Biology 63:165001.

Ghatwary N, Ye X, Zolgharni M. 2019. Esophageal abnormality detection using densenet based faster r-cnn with gabor features. IEEE Access 7:84374-84385.

Ghatwary N, Zolgharni M, Ye X. 2019. Early esophageal adenocarcinoma detection using deep learning methods. International journal of computer assisted radiology and surgery 14:611-621.

Ghosh T, Fattah SA, Wahid KA. 2018. CHOBS: Color histogram of block statistics for automatic bleeding detection in wireless capsule endoscopy video. IEEE journal of translational engineering in health and medicine 6:1-12.

Hamashima C, Shabana M, Okada K, Okamoto M, Osaki Y. 2015. Mortality reduction from gastric cancer by endoscopic and radiographic screening. Cancer science 106:1744-1749.

He J-Y, Wu X, Jiang Y-G, Peng Q, Jain R. 2018. Hookworm detection in wireless capsule endoscopy images with deep learning. IEEE Transactions on Image Processing 27:23792392.

He K, Zhang X, Ren S, Sun J. 2016. Deep Residual Learning for Image Recognition. In: IEEE Conference on Computer Vision and Pattern Recognition. DOI: 10.1109/CVPR.2016.90.

Huang G, Liu Z, Van Der Maaten L, Weinberger KQ. 2017. Densely connected convolutional networks. In: Proceedings of the IEEE conference on computer vision and pattern recognition. 4700-4708.

Igarashi S, Sasaki Y, Mikami T, Sakuraba H, Fukuda S. 2020. Anatomical classification of upper gastrointestinal organs under various image capture conditions using AlexNet. Computers in Biology and Medicine 124:103950.

Imtiaz H, Fattah SA. 2010. A DCT-based feature extraction algorithm for palm-print recognition. In: 2010 INTERNATIONAL CONFERENCE ON COMMUNICATION CONTROL AND COMPUTING TECHNOLOGIES. IEEE, 657-660.

Jin S, Wang B, Xu H, Luo C, Wei L, Zhao W, Hou X, Ma W, Xu Z, Zheng Z. 2020. AI-assisted CT imaging analysis for COVID-19 screening: Building and deploying a medical AI system in four weeks. medRxiv.

Peer] Comput. Sci. reviewing PDF | (CS-2020:10:54985:1:1:NEW 20 Jan 2021) 
856

857

858

859

860

861

862

863

864

865

866

867

868

869

870

871

872

873

874

875

876

877

878

879

880

881

882

883

884

885

886

887

888

889

890

891

892

893

894

895

Kainuma M, Furusyo N, Urita Y, Nagata M, Ihara T, Oji T, Nakaguchi T, Namiki T, Hayashi J. 2015. The association between objective tongue color and endoscopic findings: results from the Kyushu and Okinawa population study (KOPS). BMC complementary and alternative medicine 15:372.

Karargyris A, Bourbakis N. 2011. Detection of small bowel polyps and ulcers in wireless capsule endoscopy videos. IEEE Transactions on biomedical engineering 58:2777-2786.

Khan MA, Kadry S, Alhaisoni M, Nam Y, Zhang Y, Rajinikanth V, Sarfraz MS. 2020a. ComputerAided Gastrointestinal Diseases Analysis From Wireless Capsule Endoscopy: A Framework of Best Features Selection. IEEE Access 8:132850-132859.

Khan MA, Khan MA, Ahmed F, Mittal M, Goyal LM, Hemanth DJ, Satapathy SC. 2020b. Gastrointestinal diseases segmentation and classification based on duo-deep architectures. Pattern Recognition Letters 131:193-204.

Khan MA, Rashid M, Sharif M, Javed K, Akram T. 2019. Classification of gastrointestinal diseases of stomach from WCE using improved saliency-based method and discriminant features selection. Multimedia Tools and Applications 78:27743-27770.

Kim D, Cho H, Cho H. 2019. Gastric Lesion Classification Using Deep Learning Based on Fast and Robust Fuzzy C-Means and Simple Linear Iterative Clustering Superpixel Algorithms. Journal of Electrical Engineering \& Technology 14:2549-2556.

Krizhevsky A, Sutskever I, Hinton GE. 2012. Imagenet classification with deep convolutional neural networks. In: Advances in neural information processing systems. 1097-1105.

Lahmiri S, Boukadoum M. 2013. Hybrid Discrete Wavelet Transform and Gabor Filter Banks Processing for Features Extraction from Biomedical Images. Journal of Medical Engineering 2013. DOI: 10.1155/2013/104684.

Lee JH, Kim YJ, Kim YW, Park S, Choi Y, Kim YJ, Park DK, Kim KG, Chung J-W. 2019. Spotting malignancies from gastric endoscopic images using deep learning. Surgical endoscopy 33:3790-3797.

Leng J, Li T, Bai G, Dong Q, Dong H. 2016. Cube-CNN-SVM: a novel hyperspectral image classification method. In: 2016 IEEE 28th International Conference on Tools with Artificial Intelligence (ICTAI). IEEE, 1027-1034.

Li B, Meng MQ-H. 2012a. Automatic polyp detection for wireless capsule endoscopy images. Expert Systems with Applications 39:10952-10958.

Li B, Meng MQ-H. 2012b. Tumor recognition in wireless capsule endoscopy images using textural features and SVM-based feature selection. IEEE Transactions on Information Technology in Biomedicine 16:323-329.

Majid A, Khan MA, Yasmin M, Rehman A, Yousafzai A, Tariq U. 2020. Classification of stomach infections: A paradigm of convolutional neural network along with classical features fusion and selection. Microscopy research and technique 83:562-576.

Mishra S, Sharma L, Majhi B, Sa PK. 2017. Microscopic image classification using det for the detection of acute lymphoblastic leukemia (all). In: Proceedings of International Conference on Computer Vision and Image Processing. Springer, 171-180.

Peer] Comput. Sci. reviewing PDF | (CS-2020:10:54985:1:1:NEW 20 Jan 2021) 
896

897

898

899

900

901

902

903

904

905

906

907

908

909

910

911

912

913

914

915

916

917

918

919

920

921

922

923

924

925

926

927

928

929

930

931

932

933

934

935

M.M. J, Q. Z, I.U. H, S. B, A. J. 2017. Three-class mammogram classification based on descriptive CNN features. Hindawi BioMed Research International 2017. DOI: 10.1155/2017/3640901.

Nadeem S, Tahir MA, Naqvi SSA, Zaid M. 2018. Ensemble of Texture and Deep Learning Features for Finding Abnormalities in the Gastro-Intestinal Tract. In: International Conference on Computational Collective Intelligence. Springer, 469-478.

Owais M, Arsalan M, Choi J, Mahmood T, Park KR. 2019. Artificial intelligence-based classification of multiple gastrointestinal diseases using endoscopy videos for clinical diagnosis. Journal of clinical medicine 8:986.

Pei M, Wu X, Guo Y, Fujita H. 2017. Small bowel motility assessment based on fully convolutional networks and long short-term memory. Knowledge-Based Systems 121:163172.

Pogorelov K, Randel KR, Griwodz C, Eskeland SL, de Lange T, Johansen D, Spampinato C, Dang-Nguyen D-T, Lux M, Schmidt PT. 2017. Kvasir: A multi-class image dataset for computer aided gastrointestinal disease detection. In: Proceedings of the 8th ACM on Multimedia Systems Conference. 164-169.

Ragab DA, Attallah O. 2020. FUSI-CAD: Coronavirus (COVID-19) diagnosis based on the fusion of CNNs and handcrafted features. PeerJ Computer Science 6:e306.

Ragab DA, Sharkas M, Attallah O. 2019. Breast cancer diagnosis using an efficient CAD system based on multiple classifiers. Diagnostics 9:165-191.

Ragab DA, Sharkas M, Marshall S, Ren J. 2019. Breast cancer detection using deep convolutional neural networks and support vector machines. PeerJ 7:e6201.

Rashidi S, Fallah A, Towhidkhah F. 2012. Feature extraction based DCT on dynamic signature verification. Scientia Iranica 19:1810-1819.

Ravì D, Wong C, Deligianni F, Berthelot M, Andreu-Perez J, Lo B, Yang G-Z. 2016. Deep learning for health informatics. IEEE journal of biomedical and health informatics 21:421.

Redmon J, Farhadi A. 2017. YOLO9000: better, faster, stronger. In: Proceedings of the IEEE conference on computer vision and pattern recognition. 7263-7271.

Sampaio WB, Diniz EM, Silva AC, De Paiva AC, Gattass M. 2011. Detection of masses in mammogram images using CNN, geostatistic functions and SVM. Computers in Biology and Medicine 41:653-664.

Sharif M, Attique Khan M, Rashid M, Yasmin M, Afza F, Tanik UJ. 2019. Deep CNN and geometric features-based gastrointestinal tract diseases detection and classification from wireless capsule endoscopy images. Journal of Experimental \& Theoretical Artificial Intelligence:1-23.

Shi Q, Li W, Zhang F, Hu W, Sun X, Gao L. 2018. Deep CNN with multi-scale rotation invariance features for ship classification. Ieee Access 6:38656-38668.

Srivastava V, Purwar RK. 2017.A Five-Level Wavelet Decomposition and Dimensional Reduction Approach for Feature Extraction and Classification of MR and CT Scan Images. 
936

937

938

939

940

941

942

943

944

945

946

947

948

949

950

951

952

953

954

955

956

957

958

959

960

961

962

963

964

965

966

967

Available at https://www.hindawi.com/journals/acisc/2017/9571262/(accessed March 12, 2019). DOI: 10.1155/2017/9571262.

Su D, Li Y, Zhao Y, Xu R, Yuan B, Wu W. 2020. A Face Recognition Algorithm Based on DualChannel Images and VGG-cut Model. In: Journal of Physics: Conference Series. IOP Publishing, 012151.

Talo M, Baloglu UB, Y1ldırım Ö, Acharya UR. 2019. Application of deep transfer learning for automated brain abnormality classification using MR images. Cognitive Systems Research 54:176-188.

Thai LH, Hai TS, Thuy NT. 2012. Image classification using support vector machine and artificial neural network. International Journal of Information Technology and Computer Science $4: 32-38$.

Wang R, Xu J, Han TX. 2019. Object instance detection with pruned Alexnet and extended training data. Signal Processing: Image Communication 70:145-156.

Wu H, Huang Q, Wang D, Gao L. 2018. A CNN-SVM combined model for pattern recognition of knee motion using mechanomyography signals. Journal of Electromyography and Kinesiology 42:136-142.

Xue D-X, Zhang R, Feng H, Wang Y-L. 2016. CNN-SVM for microvascular morphological type recognition with data augmentation. Journal of medical and biological engineering 36:755-764.

Yuan Y, Li B, Meng MQ-H. 2015. Improved bag of feature for automatic polyp detection in wireless capsule endoscopy images. IEEE Transactions on automation science and engineering 13:529-535.

Yuan Y, Meng MQ-H. 2014. Polyp classification based on bag of features and saliency in wireless capsule endoscopy. In: 2014 IEEE International Conference on Robotics and Automation (ICRA). IEEE, 3930-3935.

Yuan Y, Meng MQ-H. 2017. Deep learning for polyp recognition in wireless capsule endoscopy images. Medical physics 44:1379-1389.

Zhang H, Wu R, Yuan T, Jiang Z, Huang S, Wu J, Hua J, Niu Z, Ji D. 2020. DE-Ada*: A novel model for breast mass classification using cross-modal pathological semantic mining and organic integration of multi-feature fusions. Information Sciences. DOI: 10.1016/j.ins.2020.05.080.

Peer] Comput. Sci. reviewing PDF | (CS-2020:10:54985:1:1:NEW 20 Jan 2021) 


\section{Figure 1}

Image samples of KASVIR dataset; (A) Dyed-Lifted-Polyp, (B) Dyed-Resection-Margin, (C) Esophagitis, (D)Normal-z-line, (E) (F) Normal-Cecum, Polyps, (G) Normal-Pylorus, and (H) Ulcerative-Colitis
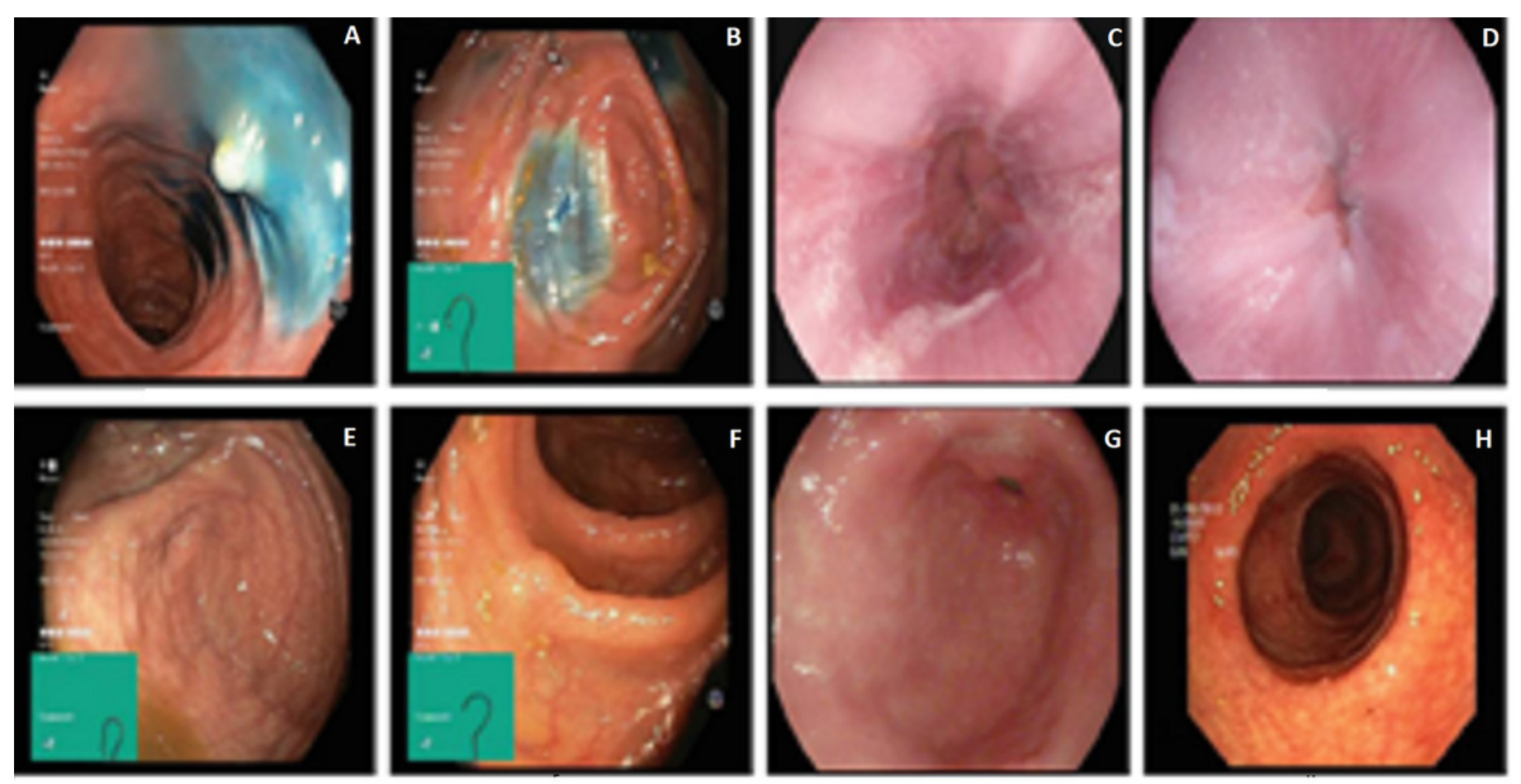


\section{Figure 2}

Image samples of Hyperkvasir dataset;

(A)bowel quality,(B) Normal Cecum, (C)Dyed-Lifted-Polyp, (D) Dyed-Resection-Margin, (E) Esophagitis, (F) Polyps, (G) Polyrous, (H) Retroflex stomach, (I) Ulcerative-Colitis, and (J)Normal-z-line
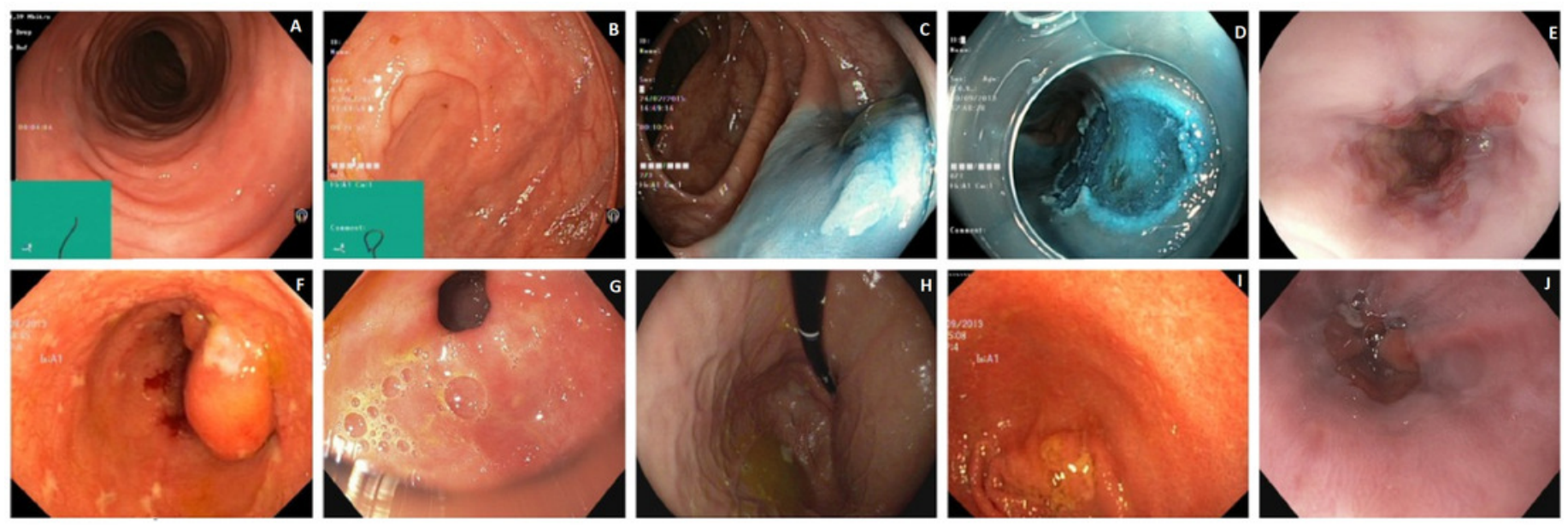
Figure 3

The block diagram of the proposed Gastro-CADx

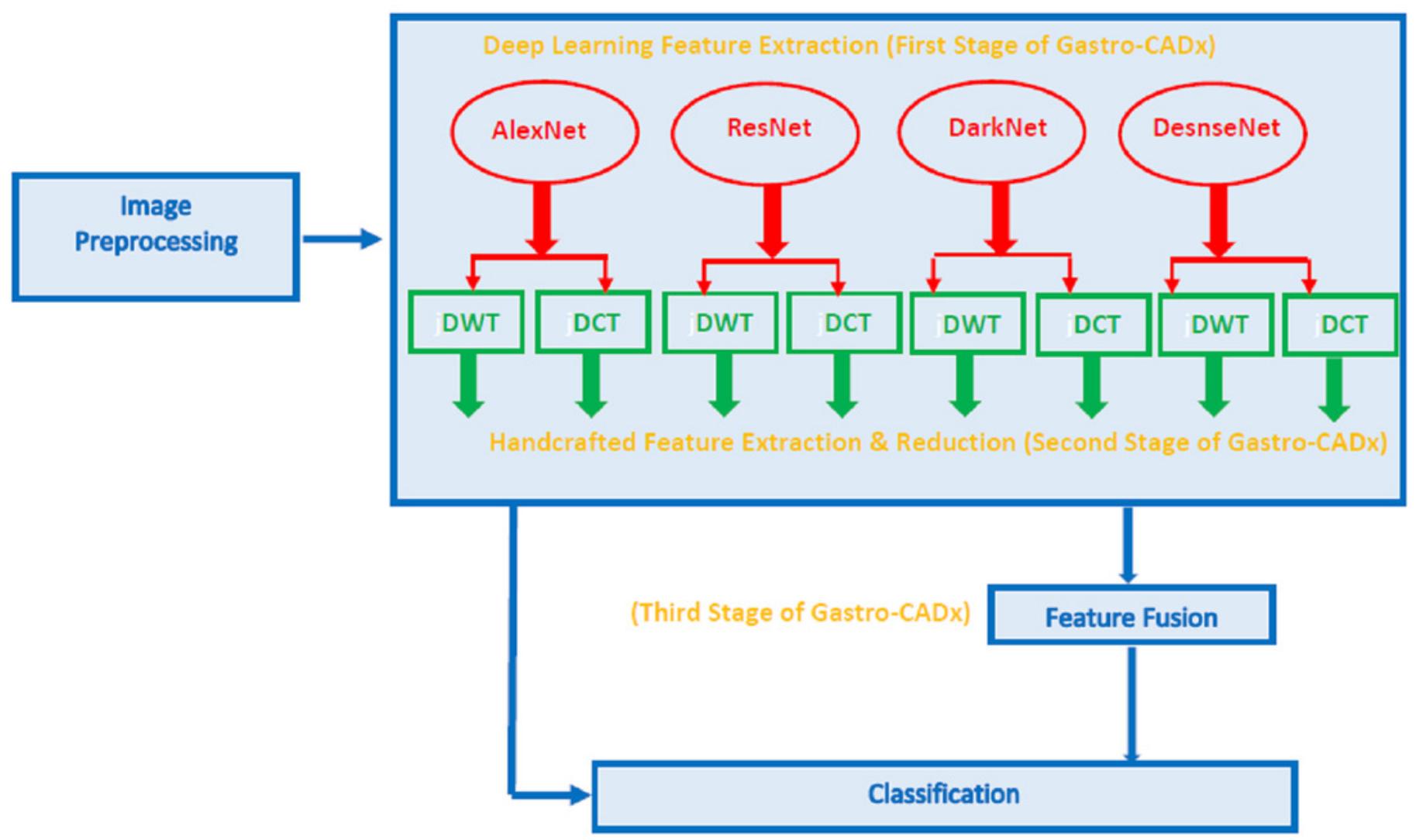


Figure 4

A summary of the four experiments of Gastro-CADx.

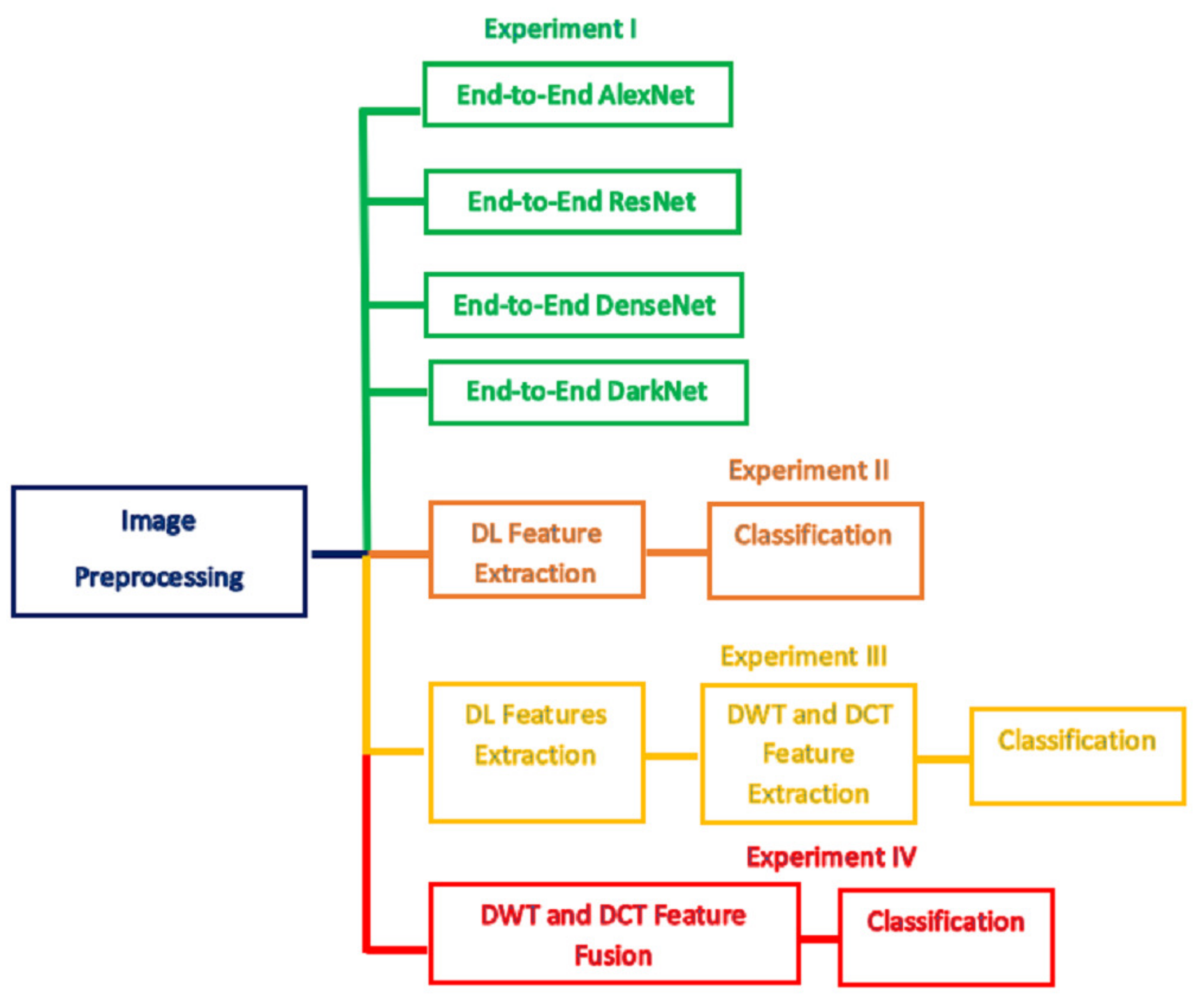


Figure 5

Experiment II - Accuracy of each DL features extracted from the four CNNs of GastroCADx constructed using Dataset I

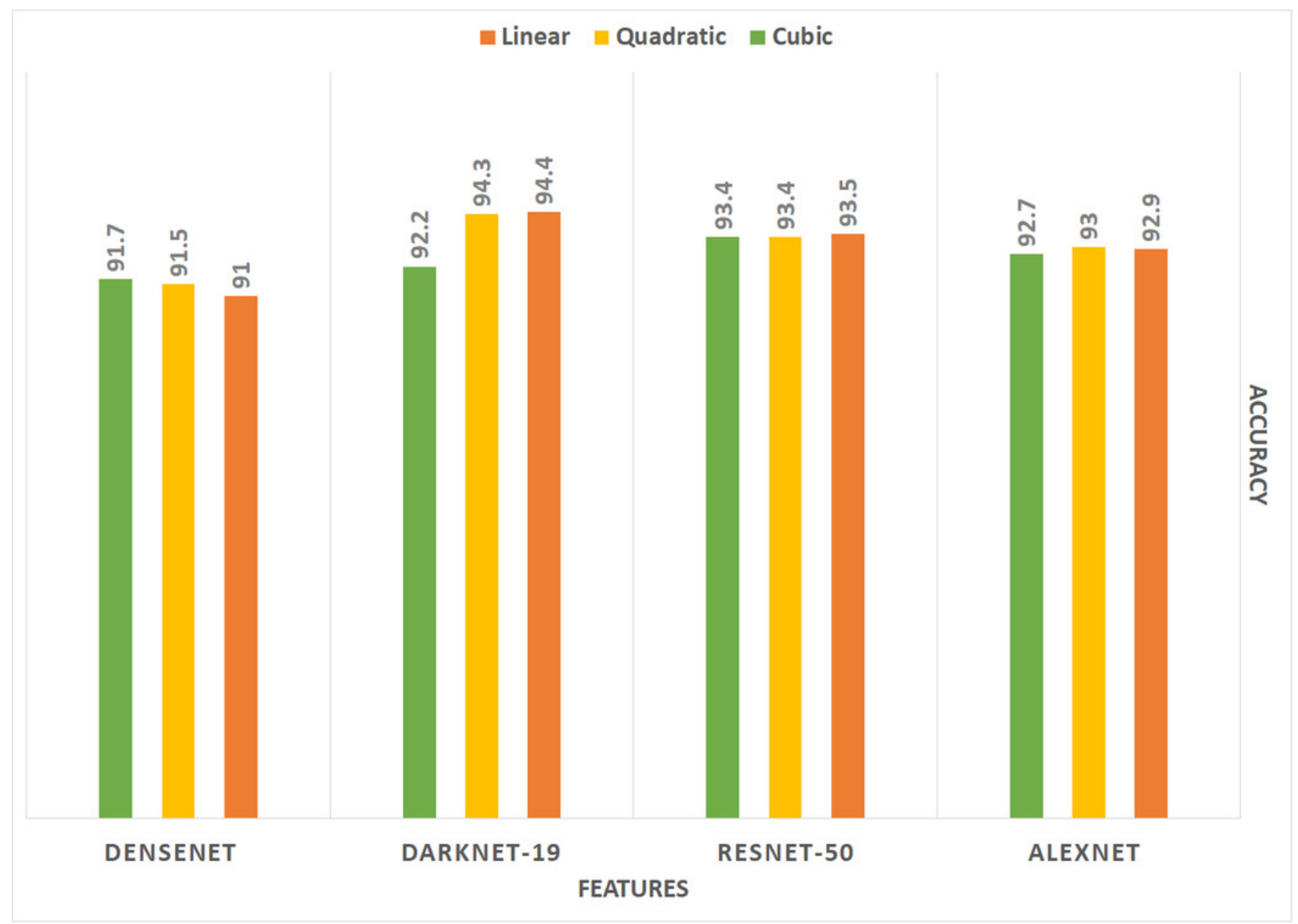


Figure 6

Experiment II - Accuracy of each DL features extracted from the four CNNs of GastroCADx constructed using Dataset II

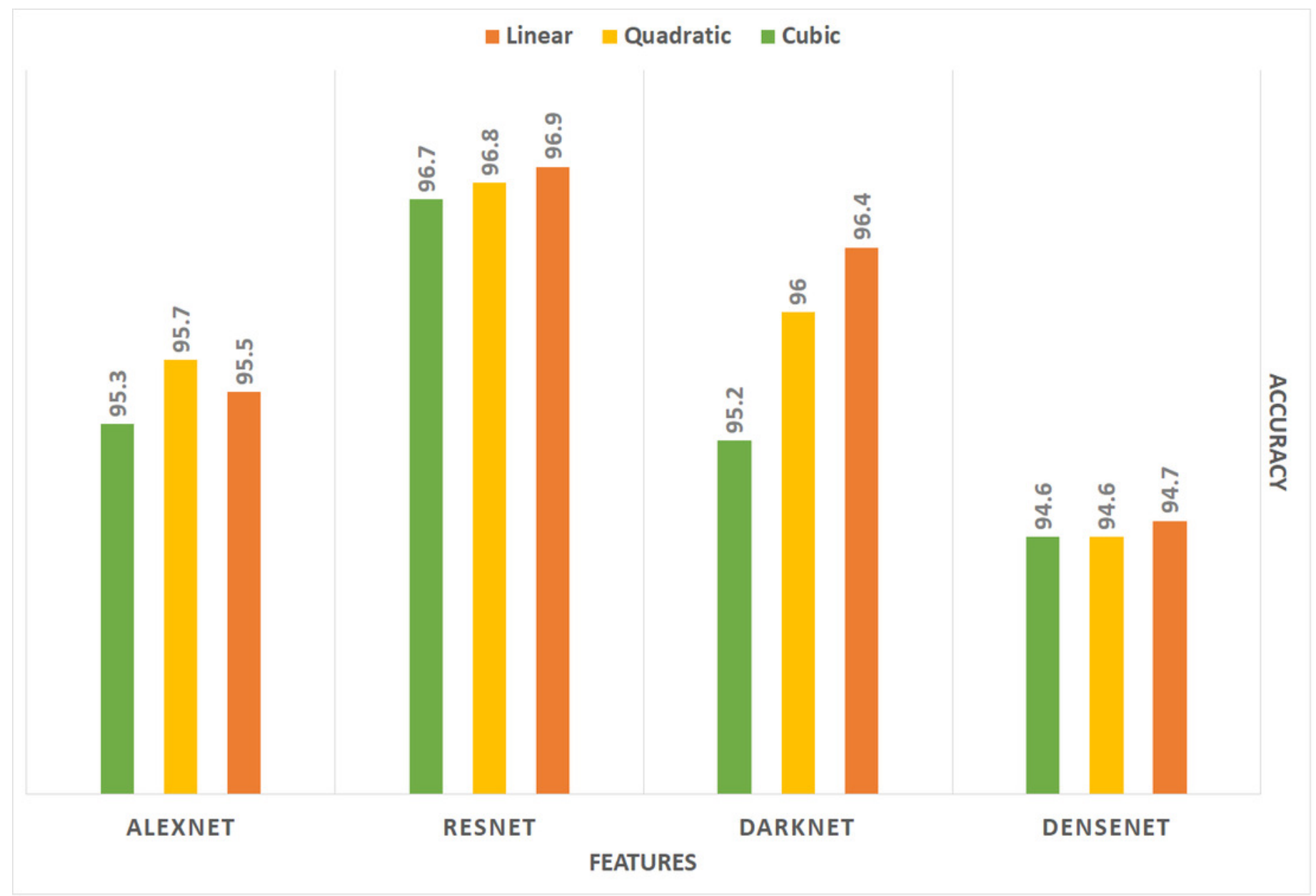




\section{Figure 7}

Experiment III - Accuracy of each DCT and DWT features extracted from the ResNet-50 CNN of Gastro-CADx constructed using Dataset I

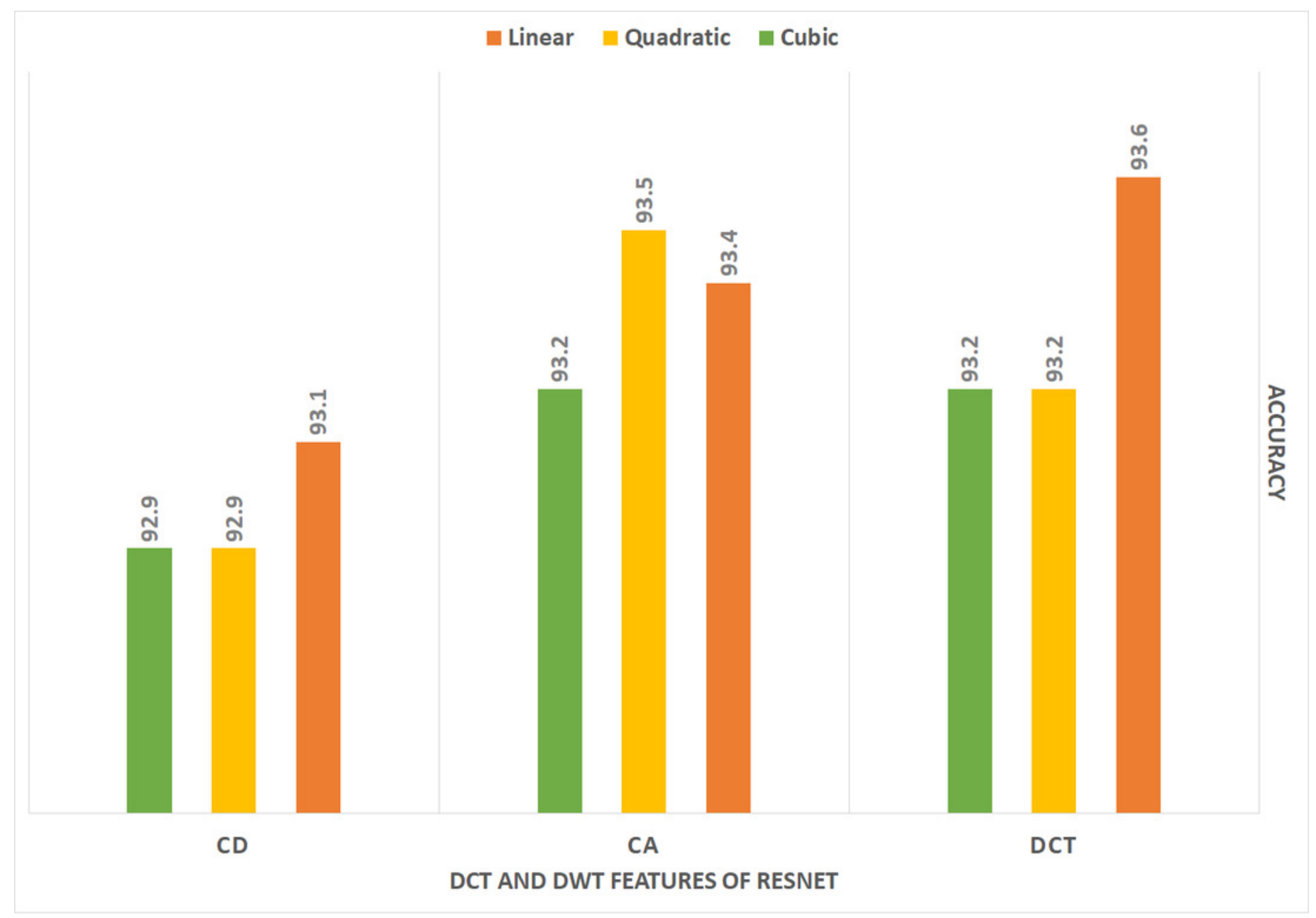


Figure 8

Experiment III - Accuracy of each DCT and DWT features extracted from the AlexNet CNN of Gastro-CADx constructed using Dataset I

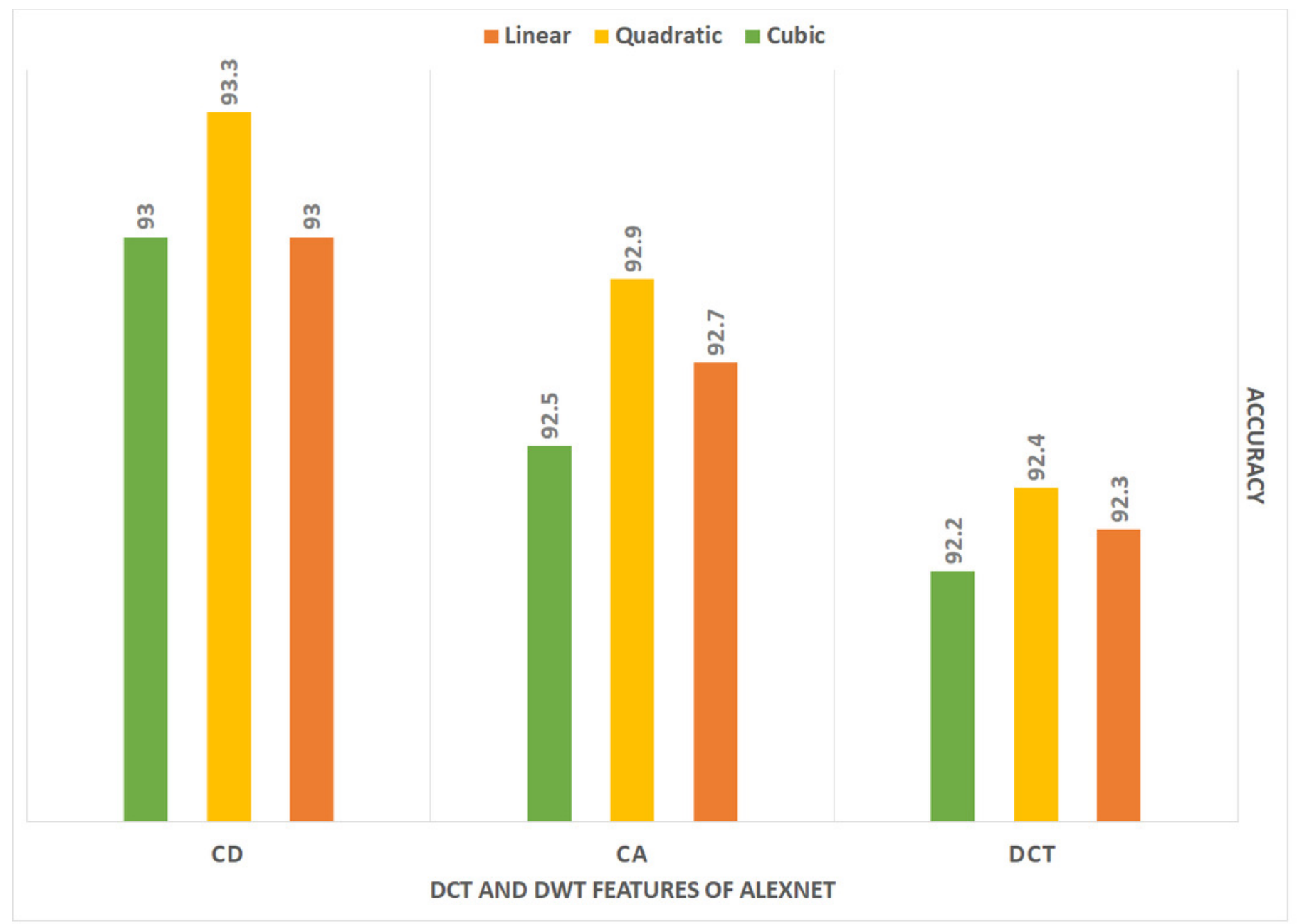


Figure 9

Experiment III - Accuracy of each DCT and DWT features extracted from the DenseNet-201 CNN of Gastro-CADx constructed using Dataset I

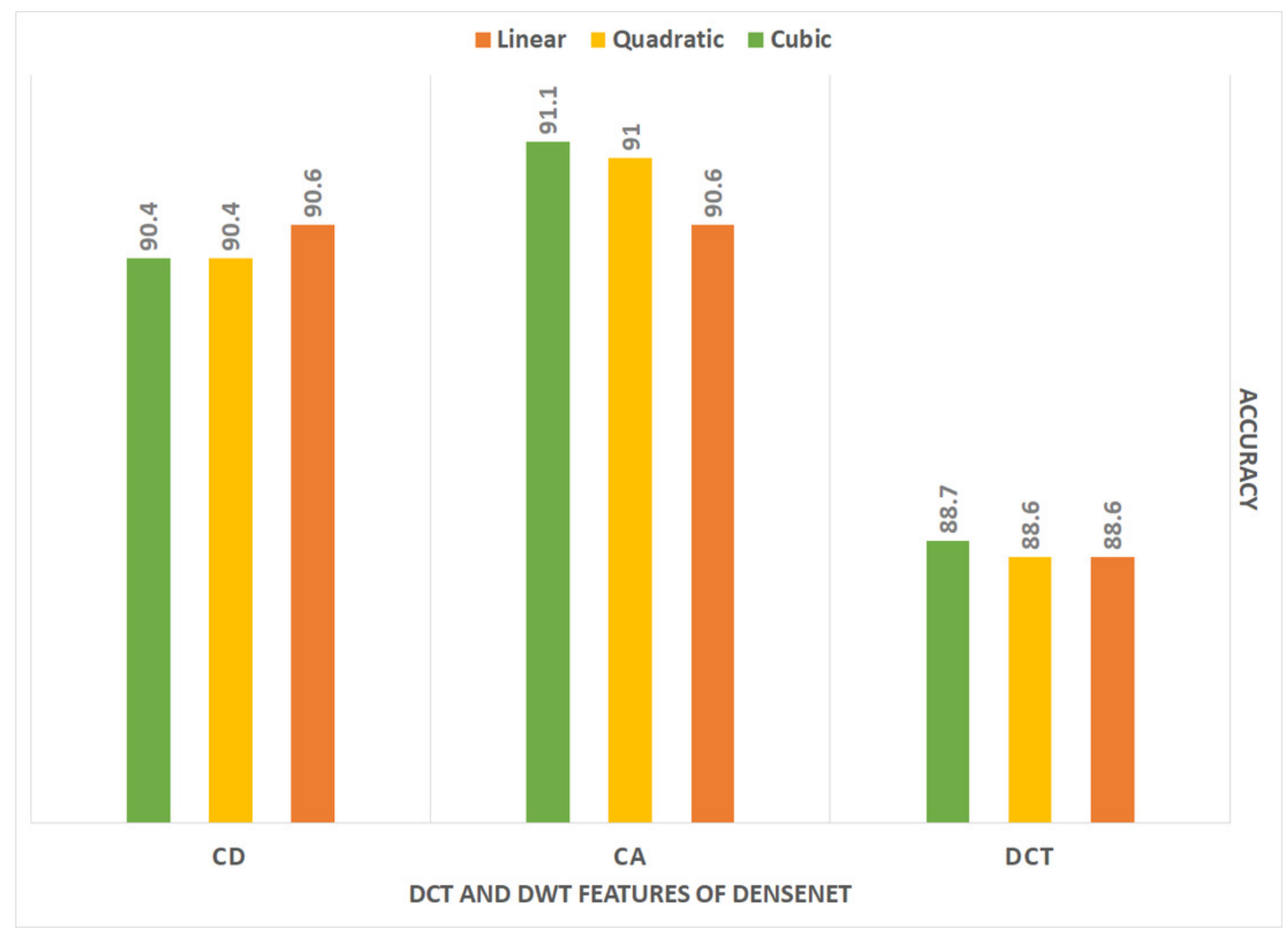




\section{Figure 10}

Experiment III - Accuracy of each DCT and DWT features extracted from the DarkNet CNN of Gastro-CADx constructed using Dataset I

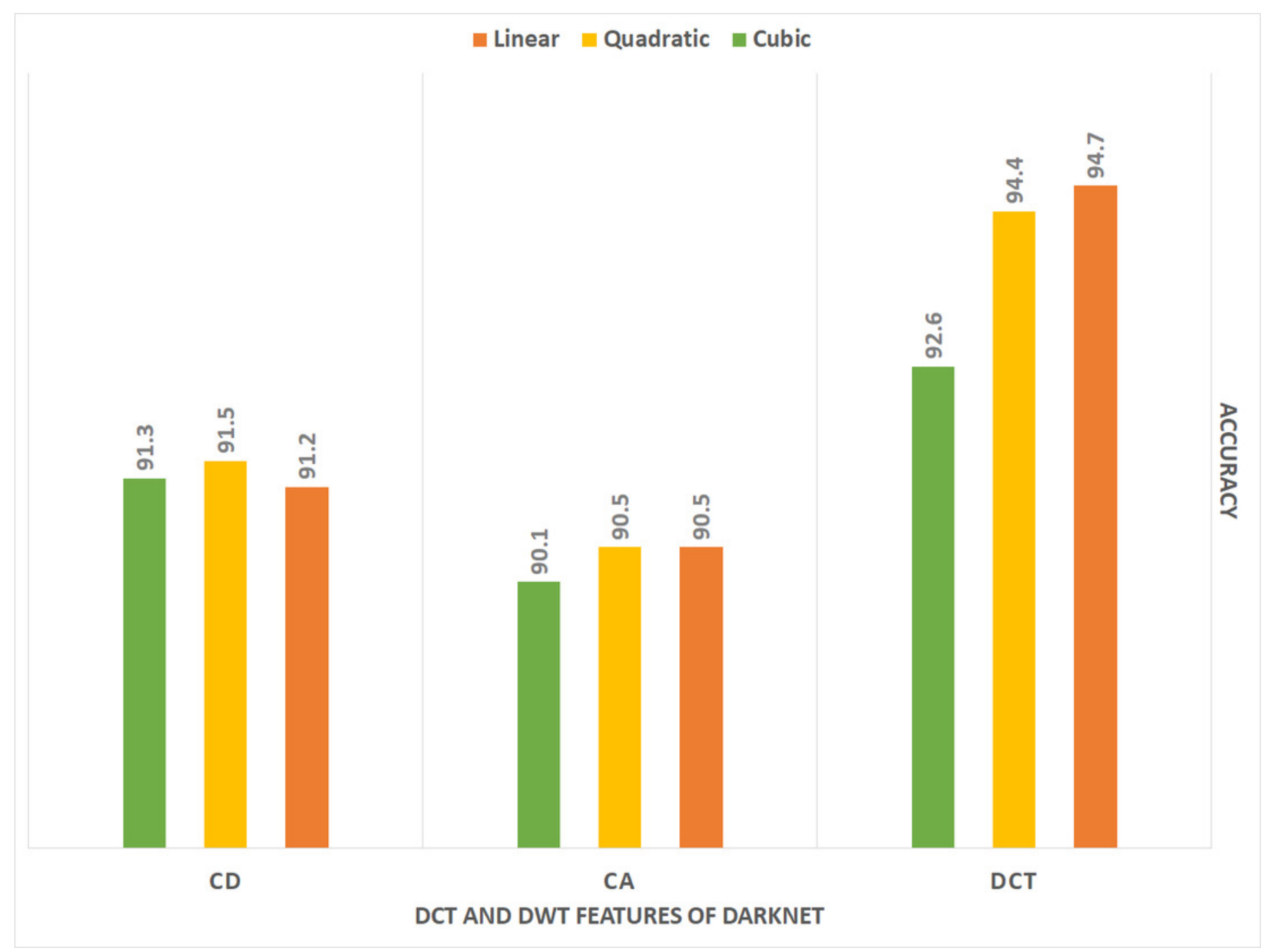


Figure 11

Experiment III - Accuracy of each DCT and DWT features extracted from the ResNet-50 CNN of Gastro-CADx constructed using Dataset II

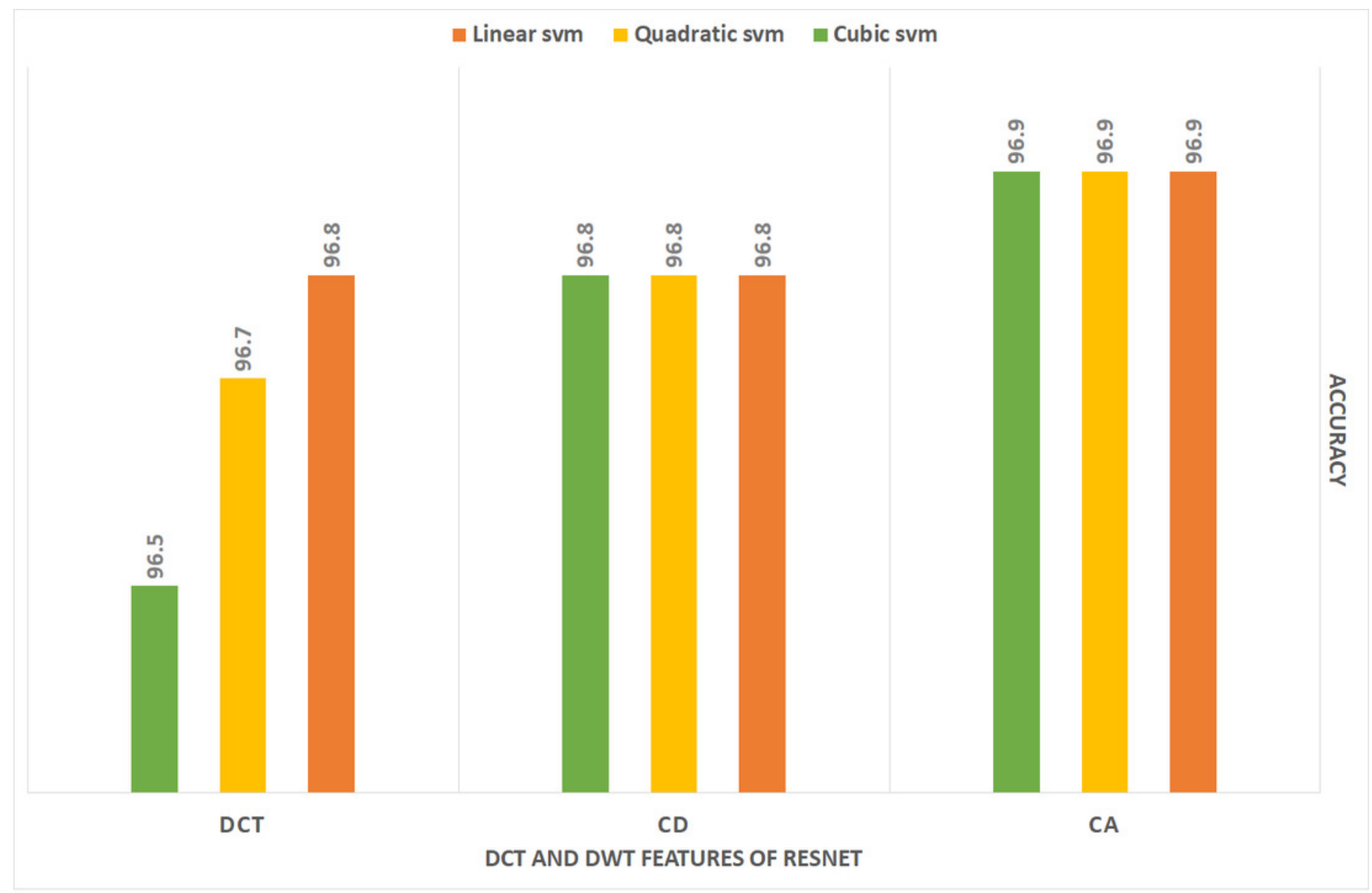


Figure 12

Experiment III - Accuracy of each DCT and DWT features extracted from the AlexNet CNN of Gastro-CADx constructed using Dataset II

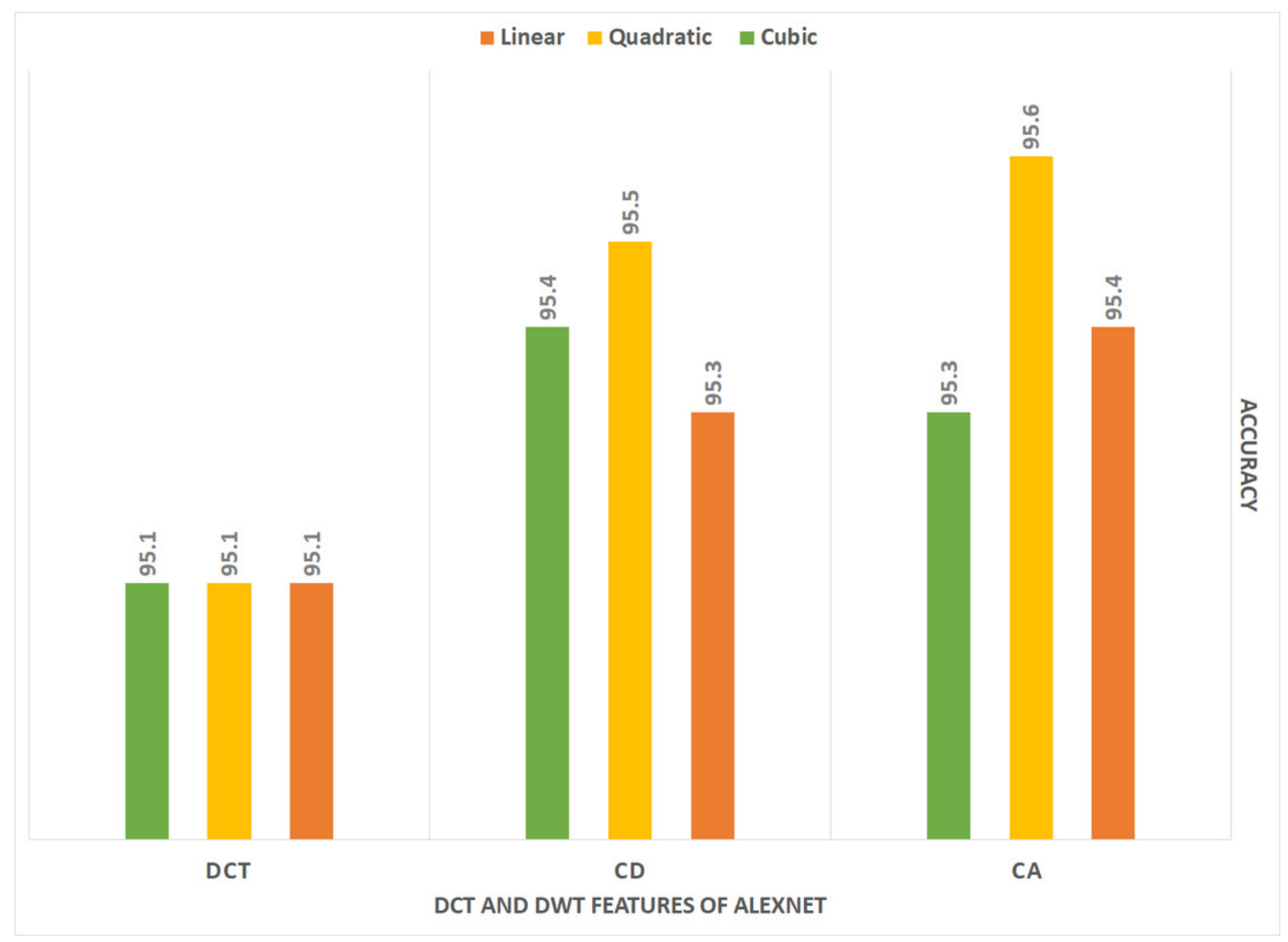


Figure 13

Experiment III - Accuracy of each DCT and DWT features extracted from the DenseNet-201 CNN of Gastro-CADx constructed using Dataset II.

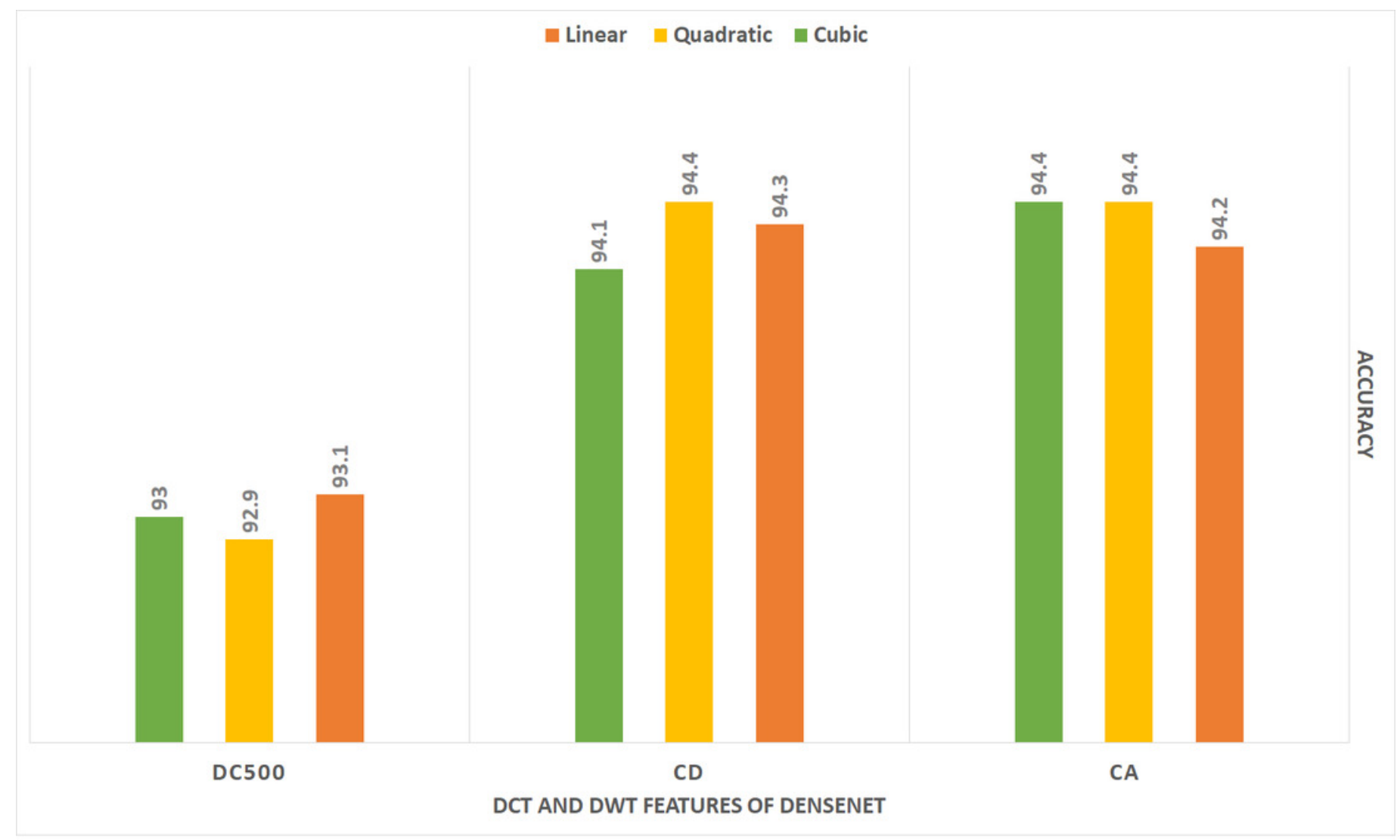


Figure 14

Experiment III - Accuracy of each DCT and DWT features extracted from the DarkNet-19 CNN of Gastro-CADx constructed using Dataset II

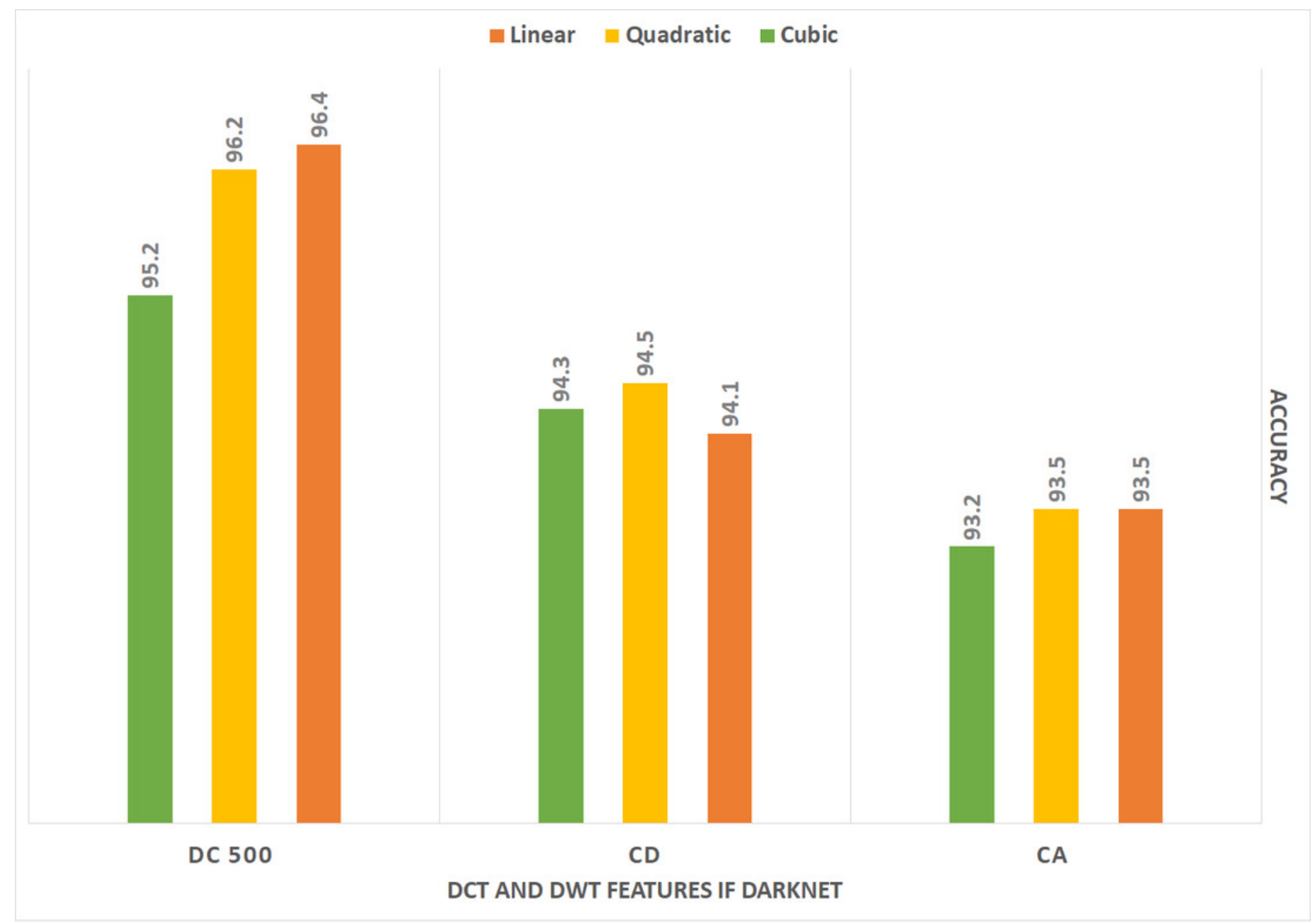


Figure 15

A comparison between the highest accuracy attained from the three stage of GastoCADx using Dataset I and II. .

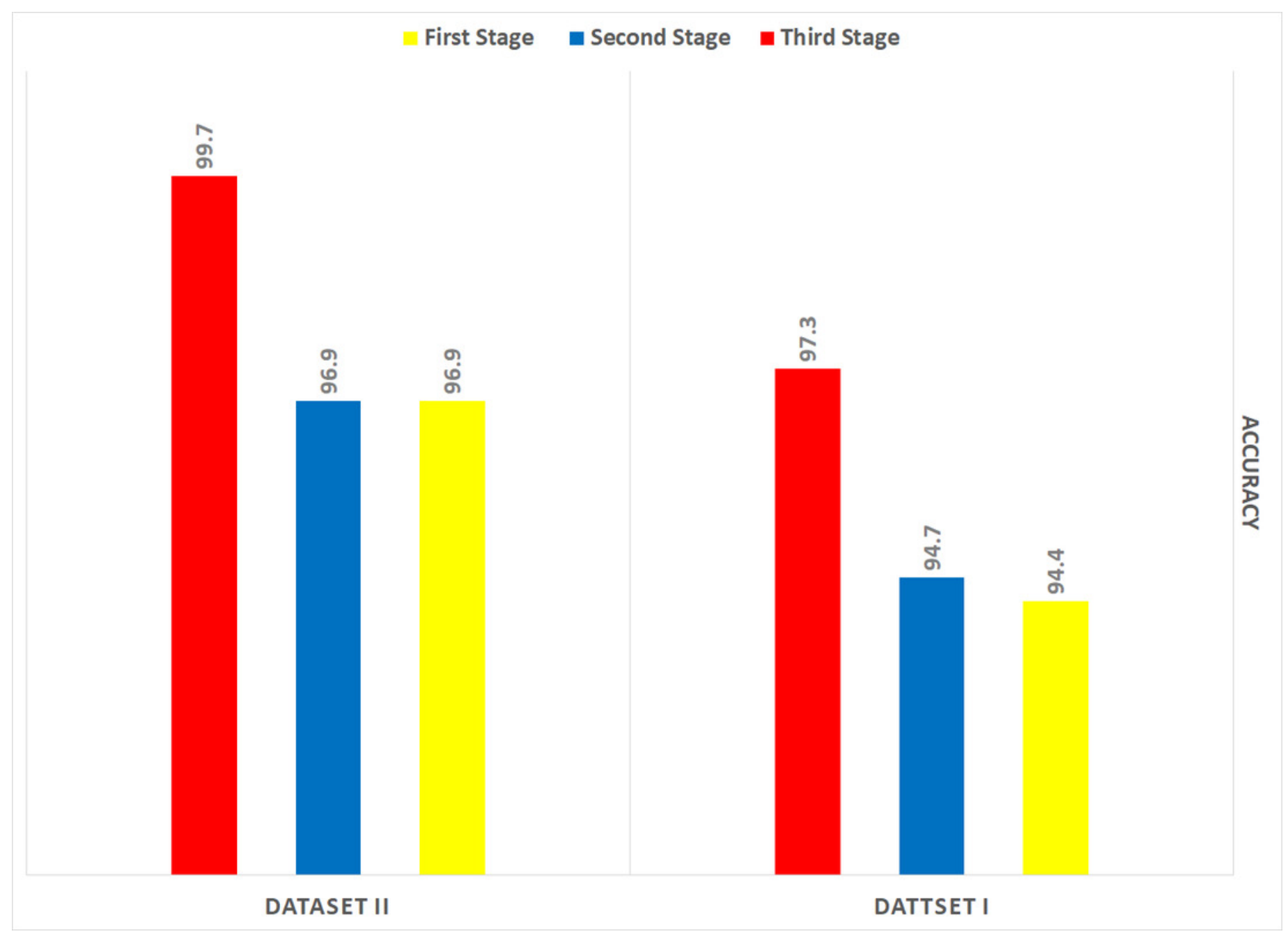




\section{Table 1 (on next page)}

A summary of recent related studies 


\begin{tabular}{|c|c|c|c|c|c|}
\hline Article & Purpose & Class & Method & Accuracy & Limitation \\
\hline $\begin{array}{l}\text { (Khan et al., } \\
\text { 2020b) }\end{array}$ & $\begin{array}{l}\text { Ulcer, polyp, } \\
\text { bleeding } \\
\text { detection }\end{array}$ & 4 & $\begin{array}{l}\text { RCNN, ResNet101, and } \\
\text { SVM }\end{array}$ & $99.13 \%$ & $\begin{array}{l}\text { - Used Only spatial } \\
\text { features. } \\
\text { - Low segmentation } \\
\text { accuracy for the } \\
\text { ulcer regions. } \\
\text { - Fail for the } \\
\text { segmentation of } \\
\text { polyp and bleeding } \\
\text { regions. }\end{array}$ \\
\hline $\begin{array}{l}\text { (Khan et al., } \\
\text { 2020a) }\end{array}$ & $\begin{array}{l}\text { Ulcer, and } \\
\text { bleeding } \\
\text { detection }\end{array}$ & 3 & VGG-16, PSO, and SVM & $98.4 \%$ & $\begin{array}{ll}\text { - } & \text { Limited classes } \\
\text { - } & \text { Used Only spatial } \\
\text { features. } \\
\text { - High Computational } \\
\text { Cost }\end{array}$ \\
\hline $\begin{array}{l}\text { (Igarashi et } \\
\text { al., 2020) }\end{array}$ & $\begin{array}{l}\text { Classify } \\
\text { several GI } \\
\text { diseases }\end{array}$ & 14 & AlexNet & $96.5 \%$ & $\begin{array}{l}\text { - Used only spatial } \\
\text { features. } \\
\text { The training or test } \\
\text { data included chosen } \\
\text { images of gastric } \\
\text { cancer lesions, } \\
\text { which could cause a } \\
\text { selection bias. } \\
\text { - Has high } \\
\text { computational cost } \\
\text { Cannot be used in } \\
\text { real-time } \\
\text { examinations }\end{array}$ \\
\hline $\begin{array}{l}\text { (Alaskar et } \\
\text { al., 2019) }\end{array}$ & $\begin{array}{l}\text { Ulcer } \\
\text { detection }\end{array}$ & 2 & AlexNet \& Google Net & $97.143 \%$ & $\begin{array}{l}\text { - Limited classes. } \\
\text { - Used only spatial } \\
\text { features }\end{array}$ \\
\hline $\begin{array}{l}\text { (Owais et al., } \\
\text { 2019) }\end{array}$ & $\begin{array}{l}\text { Classification } \\
\text { of multiple } \\
\text { GI diseases }\end{array}$ & 37 & ResNet-18 and LSTM & $89.95 \%$ & $\begin{array}{ll}- & \text { High Computational } \\
\text { Cost. } \\
\text { - } \quad \text { Used individual type } \\
\text { of features } \\
\text { - } \quad \text { Low Accuracy } \\
\end{array}$ \\
\hline $\begin{array}{l}\text { (Fan et al., } \\
\text { 2018) }\end{array}$ & $\begin{array}{l}\text { Ulcer and } \\
\text { Erosion } \\
\text { detection }\end{array}$ & 2 & AlexNet & $\begin{array}{l}95.16 \% \\
95.34 \%\end{array}$ & $\begin{array}{l}\text { - } \text { Limited classes. } \\
\text { - Used only spatial } \\
\text { features. } \\
\text { Used only one type } \\
\text { of CNN features } \\
\text { The CADx was } \\
\text { applied separately } \\
\text { for ulcer and } \\
\text { Erosion detection }\end{array}$ \\
\hline $\begin{array}{l}\text { (He et al., } \\
2018)\end{array}$ & $\begin{array}{l}\text { Hookworm } \\
\text { detection }\end{array}$ & 2 & VGG-16 and Inception & $88.5 \%$ & $\begin{array}{ll}\text { - } & \text { Limited classes. } \\
\text { - } & \text { Used only spatial } \\
& \text { features } \\
\text { - } & \text { Low accuracy } \\
\end{array}$ \\
\hline $\begin{array}{l}\text { (Yuan \& } \\
\text { Meng, 2017) }\end{array}$ & $\begin{array}{l}\text { Polyps } \\
\text { detection }\end{array}$ & 2 & $\begin{array}{l}\text { Stacked sparse auto-encoder } \\
\text { with image manifold }\end{array}$ & $98 \%$ & $\begin{array}{l}\text { - Limited classes. } \\
\text { - Used only spatial } \\
\text { features }\end{array}$ \\
\hline (Pei et al., & Bowel & 2 & LSTM and PCA & $88.8 \%$ & - $\quad$ Limited classes. \\
\hline
\end{tabular}




\begin{tabular}{|c|c|c|c|c|c|}
\hline 2017) & $\begin{array}{l}\text { detection and } \\
\text { assessment }\end{array}$ & & & & $\begin{array}{l}\text { - Used only temporal } \\
\text { features. } \\
\text { - Used only one type } \\
\text { of CNN features } \\
\text { - Low accuracy } \\
\text { - Small dataset }\end{array}$ \\
\hline $\begin{array}{l}\text { (Sharif et al., } \\
\text { 2019) }\end{array}$ & $\begin{array}{l}\text { Ulcer, and } \\
\text { bleeding } \\
\text { detection }\end{array}$ & 3 & $\begin{array}{ll}\text { VGG-16, } & \text { VGG-19, } \\
\text { geometric features, KNN }\end{array}$ & $99.42 \%$ & $\begin{array}{ll}\text { - } & \text { Limited classes. } \\
\text { - } & \text { Small dataset. } \\
\text { - Used spatial and } \\
\text { geometric features } \\
\text { only }\end{array}$ \\
\hline $\begin{array}{l}\text { (Ghatwary, } \\
\text { Ye \& } \\
\text { Zolgharni, } \\
\text { 2019) }\end{array}$ & $\begin{array}{l}\text { Esophageal } \\
\text { cancer } \\
\text { detection }\end{array}$ & 2 & $\begin{array}{l}\text { Gabor Filter. faster R-CNN, } \\
\text { and SVM }\end{array}$ & $95 \%$ & $\begin{array}{l}\text { - Limited classes. } \\
\text { - Used only one type } \\
\text { of CNN features } \\
\text { - Used spatial and } \\
\text { textural based - } \\
\text { Gabor features only. } \\
\text { - High computational } \\
\text { cost }\end{array}$ \\
\hline $\begin{array}{l}\text { Billah, } \\
\text { Waheed \& } \\
\text { Rahman, } \\
\text { 2017) }\end{array}$ & $\begin{array}{l}\text { Polyps } \\
\text { detection }\end{array}$ & 2 & $\begin{array}{l}\text { Color based DWT,CNN, } \\
\text { and SVM }\end{array}$ & $98.65 \%$ & $\begin{array}{ll}\text { - } & \text { Limited classes. } \\
\text { - } & \text { Used only one type } \\
\text { of CNN features } \\
\text { - Used spatial and } \\
\text { color based -DWT } \\
\text { only } \\
\text { - } & \text { Small dataset } \\
\end{array}$ \\
\hline $\begin{array}{l}\text { (Nadeem et } \\
\text { al., 2018) }\end{array}$ & $\begin{array}{l}\text { Classification } \\
\text { of several GI } \\
\text { diseases }\end{array}$ & 8 & $\begin{array}{l}\text { VGG-19, Haralick and LBP } \\
\text { texture analysis, and } \\
\text { logistic regression }\end{array}$ & $83 \%$ & $\begin{array}{ll}\text { - } & \text { Low accuracy } \\
\text { - } & \text { Used only one type } \\
\text { of CNN features } \\
\text { - } \\
\text { Used spatial features } \\
\text { based on CNN and } \\
\text { textural analysis } \\
\text { only }\end{array}$ \\
\hline $\begin{array}{l}\text { (Majid et al., } \\
2020 \text { ) }\end{array}$ & $\begin{array}{l}\text { Bleeding, } \\
\text { esophagitis, } \\
\text { polyp, and } \\
\text { ulcerative- } \\
\text { colitis } \\
\text { classification } \\
\end{array}$ & 5 & $\begin{array}{l}\text { DCT, color based statistical } \\
\text { features, DWT, VGG-16, } \\
\text { GA, and E }\end{array}$ & $96.5 \%$ & $\begin{array}{l}\text { - High Computational } \\
\text { cost. } \\
\text { - Used only one type } \\
\text { of CNN DL features }\end{array}$ \\
\hline $\begin{array}{l}\text { (Nguyen et } \\
\text { al., 2020) }\end{array}$ & $\begin{array}{l}\text { Classifying } \\
\text { images to } \\
\text { normal and } \\
\text { abnormal }\end{array}$ & 2 & $\begin{array}{l}\text { DenseNet, Inception, and } \\
\text { VGG-16 }\end{array}$ & $70.7 \%$ & $\begin{array}{l}\text { - Classify images to } \\
\text { either normal or } \\
\text { abnormal. } \\
\text { - Did not classify } \\
\text { several GI diseases. } \\
\text { - Low accuracy }\end{array}$ \\
\hline $\begin{array}{l}\text { (Owais et al., } \\
2020)\end{array}$ & $\begin{array}{l}\text { Classification } \\
\text { of multiple } \\
\text { GI diseases }\end{array}$ & 37 & DenseNet and LSTM & $95.75 \%$ & $\begin{array}{ll}\text { - } & \text { High Computational } \\
\text { Cost. } \\
\text { - } \\
\text { Used individual type } \\
\text { of features }\end{array}$ \\
\hline
\end{tabular}




\section{Table 2 (on next page)}

A summary of the four CNNs architectures 


\begin{tabular}{|l|l|l|l|}
\hline CNN Structure & Number of layers & Size of input & Size of output \\
\hline AlexNet & 23 & $227 \times 227$ & 4096 \\
\hline ResNet-50 & 50 & $224 \times 224$ & 2048 \\
\hline DarkNet-19 & 19 & $256 \times 256$ & 8 and 10 \\
\hline DenseNet-201 & 201 & $224 \times 224$ & 1920 \\
\hline
\end{tabular}




\section{Table 3 (on next page)}

The Classification Accuracy for the four CNNs used in Gastro-CADx using Dataset I 


\begin{tabular}{|c|c|}
\hline CNN & Accuracy (\%) \\
\hline AlexNet & 88.32 \\
\hline ResNet-50 & 91.66 \\
\hline DarkNet-19 & 90.08 \\
\hline DenseNet-201 & 89.83 \\
\hline
\end{tabular}




\section{Table 4 (on next page)}

The Classification Accuracy for the four CNNs used in Gastro-CADx using Dataset II 


\begin{tabular}{|c|c|}
\hline CNN & Accuracy (\%) \\
\hline AlexNet & 91.66 \\
\hline ResNet-50 & 94.75 \\
\hline DarkNet-19 & 93.26 \\
\hline DenseNet-201 & 91.93 \\
\hline
\end{tabular}




\section{Table 5 (on next page)}

The Classification Accuracy (\%) for the CA and CD features of DWT extracted from different combination of CNNs used in Gastro-CADx using Dataset I 


\begin{tabular}{|l|c|c|c|c|c|c|}
\hline \multirow{2}{*}{ Features } & \multicolumn{3}{c|}{ CA } & \multicolumn{3}{c|}{ CD } \\
\cline { 2 - 7 } & Linear & Quadratic & Cubic & Linear & Quadratic & Cubic \\
\hline ResNet+DarkNet & 94.8 & 95 & 95 & 93.8 & 94 & 93.7 \\
\hline AlexNet+DarkNet & 93.7 & 94.5 & 94.6 & 93.4 & 93.7 & 93.4 \\
\hline DenseNet+DarkNet & 48.8 & 48.5 & 47.6 & 48.4 & 48.6 & 47.7 \\
\hline AlexNet+ResNet & 94.2 & 94.4 & 94.1 & 94.3 & 94.7 & 94.5 \\
\hline AlexNet+DenseNet & 95.7 & 96 & 96.2 & 95.8 & 96.2 & 96 \\
\hline ResNet +DenseNet & 96.1 & 96.3 & 96.6 & 96.4 & 96.5 & 96.7 \\
\hline AlexNet+ResNet +DenseNet & 96.8 & $\mathbf{9 7 . 3}$ & $\mathbf{9 7 . 3}$ & $\mathbf{9 6 . 5}$ & 96.8 & $\mathbf{9 6 . 8}$ \\
\hline AlexNet+ResNet +DarkNet & 95.2 & 95.3 & 95.4 & 94.4 & 94.7 & 94.7 \\
\hline AlexNet+ DenseNet +DarkNet & 95.6 & 95.9 & 96.2 & 95.9 & 96.4 & 96.3 \\
\hline ResNet +DenseNet+DarkNet & 96.3 & 96.7 & 96.7 & 96.3 & 96.4 & 96.5 \\
\hline AlexNet+ResNet +DenseNet+DarkNet & 96.6 & 96.8 & 97 & 96.3 & 96.6 & 96.6 \\
\hline
\end{tabular}




\section{Table 6(on next page)}

The Classification Accuracy (\%) for the 500 DCT features extracted from different combination of CNNs used in Gastro-CADx using Dataset I 


\begin{tabular}{|l|c|c|c|}
\hline \multicolumn{1}{|c|}{ Features } & Linear & Quadratic & Cubic \\
\hline ResNet+DarkNet & 95 & 95 & 95.3 \\
\hline AlexNet+DarkNet & 95 & 95 & 95.1 \\
\hline DenseNet+DarkNet & 47.7 & 47.5 & 46.6 \\
\hline AlexNet+ResNet & 94.2 & 94.2 & 94.4 \\
\hline AlexNet+DenseNet & 95.5 & 95.7 & 96.1 \\
\hline ResNet +DenseNet & 96.2 & 96.2 & 96.3 \\
\hline AlexNet+ResNet +DarkNet & 95.5 & 95.8 & 95.9 \\
\hline AlexNet+ DenseNet +DarkNet & 95.8 & 95.9 & 96 \\
\hline ResNet +DenseNet+DarkNet & 96.3 & 96.5 & 96.5 \\
\hline AlexNet+ResNet +DenseNet & 96.8 & 97 & 97.1 \\
\hline AlexNet+ResNet +DenseNet+DarkNet & $\mathbf{9 7 . 1}$ & $\mathbf{9 7 . 3}$ & $\mathbf{9 7 . 3}$ \\
\hline
\end{tabular}




\section{Table 7 (on next page)}

The Classification Accuracy (\%) for the CA and CD features of DWT extracted from different combination of CNNs used in Gastro-CADx using Dataset II 


\begin{tabular}{|l|r|r|r|r|r|r|}
\hline \multirow{2}{*}{ Features } & \multicolumn{3}{c|}{ CA } & \multicolumn{3}{c|}{ CD } \\
\cline { 2 - 7 } & Linear & Quadratic & Cubic & Linear & Quadratic & Cubic \\
\hline ResNet+DarkNet & 98.6 & 98.6 & 98.6 & 98.4 & 98.6 & 98.6 \\
\hline AlexNet+DarkNet & 98.6 & 98.7 & 98.9 & 98.1 & 98.6 & 98.7 \\
\hline DenseNet+DarkNet & 97.1 & 97.3 & 97.3 & 96.5 & 96.7 & 96.7 \\
\hline AlexNet+ResNet & 98.7 & 99 & 99 & 98.6 & 98.9 & 99 \\
\hline AlexNet+DenseNet & 97.7 & 98 & 98.1 & 97.7 & 98 & 98.1 \\
\hline ResNet +DenseNet & 48.8 & 49 & 49.2 & 48.7 & 49 & 49.1 \\
\hline AlexNet+ResNet +DenseNet & 99.6 & 99.6 & 99.7 & 99.5 & 99.6 & 99.5 \\
\hline AlexNet+ResNet +DarkNet & 98.8 & 98.8 & 98.9 & 98.8 & 98.9 & 98.9 \\
\hline AlexNet+ DenseNet +DarkNet & 98.7 & 98.7 & 98.8 & 98.6 & 98.8 & 98.8 \\
\hline ResNet +DenseNet+DarkNet & 98.8 & 98.9 & 99 & 98.7 & 99 & 99.1 \\
\hline AlexNet+ResNet +DenseNet+DarkNet & 99.6 & 99.6 & 99.6 & 99.4 & 99.5 & 99.6 \\
\hline
\end{tabular}




\section{Table 8 (on next page)}

The Classi $\square$ cation Accuracy (\%) for the 500 DCT features extracted from di $\square$ erent combination of CNNs used in Gastro-CADx using Dataset II 


\begin{tabular}{|l|r|r|r|}
\hline \multicolumn{1}{|c|}{ Features } & \multicolumn{1}{c|}{ Linear } & Quadratic & \multicolumn{1}{c|}{ Cubic } \\
\hline ResNet+DarkNet & 98.1 & 98.4 & 98.5 \\
\hline AlexNet+DarkNet & 97.7 & 97.9 & 98 \\
\hline DenseNet+DarkNet & 48.7 & 49 & 49.2 \\
\hline AlexNet+ResNet & 98.2 & 98.5 & 98.6 \\
\hline AlexNet+DenseNet & 98.2 & 98.6 & 98.6 \\
\hline ResNet +DenseNet & 98.8 & 98.9 & 98.9 \\
\hline AlexNet+ResNet +DarkNet & 98.8 & 99 & 99.2 \\
\hline AlexNet+ DenseNet +DarkNet & 98.6 & 98.7 & 98.7 \\
\hline ResNet +DenseNet+DarkNet & 98.6 & 98.9 & 98.9 \\
\hline AlexNet+ResNet +DenseNet & 99.6 & 99.6 & 99.6 \\
\hline AlexNet+ResNet +DenseNet+DarkNet & 98.7 & 99.5 & 99.6 \\
\hline
\end{tabular}




\section{Table 9 (on next page)}

The Performance metrics for the CA features of DWT extracted from AlexNet+ResNet +DenseNet CNNs using Dataset I and II. 


\begin{tabular}{|c|c|c|c|c|}
\hline & Specificity & Sensitivity & Precision & F1 score \\
\hline \multicolumn{5}{|c|}{ Dataset I } \\
\hline Cubic SVM & 0.9959 & 0.9715 & 0.9718 & 0.9715 \\
\hline \multicolumn{5}{|c|}{ Dataset I } \\
\hline Cubic SVM & 0.9996 & 0.9965 & 0.9961 & 0.9963 \\
\hline
\end{tabular}




\section{Table $\mathbf{1 0}$ (on next page)}

The classi $\square$ cation Accuracy (\%) for the $\square$ rst stage of Gastro-CADx compared to Experiment I (end-to-end classi $\square$ ction process) using Dataset I and II 


\begin{tabular}{|c|c|c|c|c|}
\hline \multirow[t]{2}{*}{ CNN } & \multirow{2}{*}{$\begin{array}{l}\text { Experiment I } \\
\text { (END to END) }\end{array}$} & \multicolumn{3}{|c|}{ First Stage of Gastro-CADx } \\
\hline & & Linear & Quadratic & Cubic \\
\hline \multicolumn{5}{|c|}{ DataSet I } \\
\hline AlexNet & 88.32 & 92.9 & 93 & 92.7 \\
\hline ResNet-50 & 91.66 & 93.5 & 93.4 & 93.4 \\
\hline DarkNet-19 & 90.08 & 94.4 & 94.3 & 92.2 \\
\hline DenseNet-201 & 89.83 & 91 & 91.5 & 91.7 \\
\hline \multicolumn{5}{|c|}{ DataSet II } \\
\hline AlexNet & 91.66 & 95.5 & 95.7 & 95.3 \\
\hline ResNet-50 & 94.75 & 96.9 & 96.8 & 96.7 \\
\hline DarkNet-19 & 93.26 & 96.4 & 96 & 95.2 \\
\hline DenseNet-201 & 91.93 & 94.7 & 94.6 & 94.6 \\
\hline
\end{tabular}




\section{Table $\mathbf{1 1}$ (on next page)}

Computation time and accuracy acheived using Gastro-CADx compared to ResNet endto-end deep learning method. 


\begin{tabular}{|c|c|c|c|c|}
\hline \multirow{2}{*}{ Method } & \multicolumn{2}{|c|}{ Dataset I } & \multicolumn{2}{c|}{ Dataset II } \\
\cline { 2 - 5 } & Training Time (sec) & Accuracy (\%) & Training Time (sec) & Accuracy (\%) \\
\hline ResNet (end-to end) & 80,580 & 90.08 & 100,800 & 94.75 \\
\hline Gastro-CADx & 210 & 97.3 & 780 & 99.7 \\
\hline
\end{tabular}

1 


\section{Table 12 (on next page)}

Comparisons with recent related studies based on Dataset I 


\begin{tabular}{|c|c|c|c|c|c|c|}
\hline \multirow{2}{*}{ Article } & \multirow[t]{2}{*}{ Method } & \multicolumn{5}{|c|}{ Performance metrics } \\
\hline & & Accuracy(\%) & Sensitivity & Precision & Specificity & F1 score \\
\hline (Ahmad et al., 2017) & AlexNet & 75.4 & - & - & - & - \\
\hline $\begin{array}{c}\text { (Agrawal et al., } \\
\text { 2017) }\end{array}$ & $\begin{array}{l}\mathrm{GF}^{1}+\text { Inception } \\
\mathrm{V} 3+\mathrm{VGG}+\mathrm{SVM}\end{array}$ & 96.1 & 0.852 & 0.847 & 0.978 & 0.827 \\
\hline $\begin{array}{c}\text { (Pogorelov et al., } \\
\text { 2017) }\end{array}$ & ResNet+LMT² & 95.7 & 0.826 & 0.829 & 0.975 & 0.802 \\
\hline $\begin{array}{c}\text { (Pogorelov et al., } \\
\text { 2017) }\end{array}$ & GF+ Decision Tree & 93.7 & 0.748 & 0.748 & 0.964 & 0.747 \\
\hline $\begin{array}{c}\text { (Pogorelov et al., } \\
\text { 2017) }\end{array}$ & GF+ Random Forest & 93.3 & 0.732 & 0.732 & 0.962 & 0.727 \\
\hline $\begin{array}{c}\text { (Nadeem et al., } \\
\text { 2018) }\end{array}$ & $\begin{array}{c}\mathrm{GF}+\mathrm{LBP}^{3}+\text { Haralick }+ \\
\mathrm{LR}\end{array}$ & 94.2 & 0.774 & 0.767 & 0.966 & 0.707 \\
\hline $\begin{array}{c}\text { (Thambawita et al., } \\
\text { 2018) }\end{array}$ & $\mathrm{GF}+\mathrm{CNN}$ & 95.8 & 0.958 & 0.9587 & 0.9971 & 0.9580 \\
\hline (Owais et al., 2019) & $\begin{array}{c}\text { ResNet+DenseNet+ML } \\
\mathrm{P}^{5}\end{array}$ & 92.5 & 0.993 & 0.946 & - & 0.934 \\
\hline Gast & -CADx & 97.3 & 0.9715 & 0.9718 & 0.9959 & 0.9715 \\
\hline
\end{tabular}

${ }^{1}$ Global Features, ${ }^{2}$ logistic model tree, ${ }^{3}$ Local Binary Pattern, ${ }^{4}$ Logistic Regression, ${ }^{5}$ Multilayer perceptron 1 


\section{Table $\mathbf{1 3}$ (on next page)}

Comparisons with studies based on Dataset II 


\begin{tabular}{|c|c|}
\hline Method & Accuracy (\%) \\
\hline AlexNet & 91.66 \\
\hline ResNet-50 (Borgli et al., 2020) & 94.75 \\
\hline DarkNet-19 & 93.26 \\
\hline DenseNet-201 & 91.93 \\
\hline Gastro-CADx & 99.7 \\
\hline
\end{tabular}

